\title{
Large deviations for invariant measures of stochastic reaction-diffusion systems with multiplicative noise and non-Lipschitz reaction term
}

\author{
Sandra Cerrai ${ }^{\mathrm{a}, *}$, Michael Röckner ${ }^{\mathrm{b}}$ \\ a Dip. di Matematica per le Decisioni, Università di Firenze, Via C. Lombroso 6/17, 50134 Firenze, Italy \\ ${ }^{\mathrm{b}}$ Fakultät für Mathematik, Universität Bielefeld, 33501 Bielefeld, Germany
}

Received 15 May 2003; accepted 30 March 2004

Available online 11 September 2004

\begin{abstract}
In this paper we prove a large deviations principle for the invariant measures of a class of reaction-diffusion systems in bounded domains of $\mathbb{R}^{d}, d \geqslant 1$, perturbed by a noise of multiplicative type. We consider reaction terms which are not Lipschitzcontinuous and diffusion coefficients in front of the noise which are not bounded and may be degenerate. This covers for example the case of Ginzburg-Landau systems with unbounded and possibly degenerate multiplicative noise.
\end{abstract}

(C) 2004 Elsevier SAS. All rights reserved.

\section{Résumé}

Dans cet article on prouve un principe de grandes déviations pour les mesures invariantes de systèmes de réaction-diffusion stochastiques dans des domaines bornés de $\mathbb{R}^{d}, d \geqslant 1$, perturbés par un bruit multiplicatif. On considère des termes de réaction qui ne sont pas lipschitziens et des coefficients de diffusion qui ne sont pas bornés et peuvent être dégénérés. Ceci s'applique par exemple au cas de systèmes de Ginzburg-Landau avec bruit multiplicatif non borné et éventuellement dégénéré.

(c) 2004 Elsevier SAS. All rights reserved.

MSC: $60 \mathrm{~F} 10 ; 60 \mathrm{H} 15$

Keywords: Large deviations principle; Stochastic partial differential equations; Invariant measures; Multiplicative noise

\footnotetext{
${ }^{*}$ Corresponding author.

E-mail addresses: sandra.cerrai@dmd.unifi.it (S.Cerrai), roeckner@mathematik.uni-bielefeld.de (M. Röckner).
} 


\section{Introduction}

In this paper we are dealing with the long-term behavior of the stochastic reaction-diffusion system

$$
\left\{\begin{array}{l}
\frac{\partial u_{i}}{\partial t}(t, \xi)=\mathcal{A}_{i} u_{i}(t, \xi)+f_{i}\left(\xi, u_{1}(t, \xi), \ldots, u_{r}(t, \xi)\right) \\
\quad+\varepsilon \sum_{j=1}^{r} g_{i j}\left(\xi, u_{1}(t, \xi), \ldots, u_{r}(t, \xi)\right) Q_{j} \frac{\partial w_{j}}{\partial t}(t, \xi), \quad t \geqslant 0, \quad \xi \in \overline{\mathcal{O}}, \\
u_{i}(0, \xi)=x_{i}(\xi), \quad \xi \in \overline{\mathcal{O}}, \quad \mathcal{B}^{i} u_{i}(t, \xi)=0, \quad t \geqslant 0, \quad \xi \in \partial \mathcal{O}, \quad 1 \leqslant i \leqslant r,
\end{array}\right.
$$

with $\varepsilon>0$. Here $\mathcal{O}$ is a bounded open set of $\mathbb{R}^{d}$, with $d \geqslant 1$, having a $C^{\infty}$ boundary. For each $i=1, \ldots, r$

$$
\mathcal{A}_{i}(\xi, D)=\sum_{h, k=1}^{d} \frac{\partial}{\partial \xi_{h}}\left(a_{h k}^{i}(\xi) \frac{\partial}{\partial \xi_{k}}\right)-\alpha_{i}, \quad \xi \in \overline{\mathcal{O}} .
$$

The constants $\alpha_{i}$ are strictly positive, the coefficients $a_{h k}^{i}$ are taken in $C^{\infty}(\overline{\mathcal{O}})$ and the matrices $a^{i}(\xi):=\left[a_{h k}^{i}(\xi)\right]_{h k}$ are non-negative and symmetric, for each $\xi \in \overline{\mathcal{O}}$, and fulfill a uniform ellipticity condition, that is

$$
\inf _{\xi \in \overline{\mathcal{O}}}\left\langle a^{i}(\xi) h, h\right\rangle \geqslant \lambda_{i}|h|^{2}, \quad h \in \mathbb{R}^{d},
$$

for some positive constants $\lambda_{i}$. Finally, the operators $\mathcal{B}^{i}$ act on $\partial \mathcal{O}$ and are assumed either of Dirichlet or of co-normal type.

The mapping $f:=\left(f_{1}, \ldots, f_{r}\right): \overline{\mathcal{O}} \times \mathbb{R}^{r} \rightarrow \mathbb{R}^{r}$ is only locally Lipschitz-continuous and has polynomial growth. The mapping $g:=\left[g_{i j}\right]: \overline{\mathcal{O}} \times \mathbb{R}^{r} \rightarrow \mathcal{L}\left(\mathbb{R}^{r}\right)$ is Lipschitz-continuous, without any assumption of boundedness and non-degeneracy.

The linear operators $Q_{j}$ are bounded on $L^{2}(\mathcal{O})$ and may be taken to be equal to the identity operator in case of space dimension $d=1$. The noisy perturbations $\partial w_{j} / \partial t$ are independent cylindrical Wiener processes on a stochastic basis $\left(\Omega, \mathcal{F}, \mathcal{F}_{t}, \mathbb{P}\right)$.

For example, in the case of space dimension $d=1$ and $r=2$, we can deal with systems of the following type

$$
\left\{\begin{array}{l}
\frac{\partial u_{1}}{\partial t}=\frac{\partial}{\partial \xi}\left(a_{1} \frac{\partial u_{1}}{\partial \xi}\right)-\alpha_{1} u_{1}-c_{1} u_{1}^{2 k+1}+f_{1}\left(u_{1}, u_{2}\right)+\left\langle g_{1}\left(u_{1}, u_{2}\right), \frac{\partial w}{\partial t}\right\rangle \\
\frac{\partial u_{2}}{\partial t}=\frac{\partial}{\partial \xi}\left(a_{2} \frac{\partial u_{2}}{\partial \xi}\right)-\alpha_{2} u_{2}-c_{2} u_{2}^{2 k+1}+f_{2}\left(u_{1}, u_{2}\right)+\left\langle g_{2}\left(u_{1}, u_{2}\right), \frac{\partial w}{\partial t}\right\rangle, \\
u_{i}(0, \xi)=x_{i}(\xi), \quad \xi \in \mathcal{O}, \quad u_{i}(t, \xi)=\eta_{i} u_{i}(t, \xi)+\left(1-\eta_{i}\right) \frac{\partial u_{i}}{\partial \xi}(t, \xi), \quad \xi \in \partial \mathcal{O},
\end{array}\right.
$$

where $a_{i}$ are positive functions in $C^{1}(\overline{\mathcal{O}}), \eta_{i} \in\{0,1\}, \alpha_{i}$ and $c_{i}$ are positive constants (in fact $\alpha_{i}$ can be taken zero in the case of Dirichlet boundary conditions, that is if $\left.\eta_{i}=1\right), f=\left(f_{1}, f_{2}\right): \mathbb{R}^{2} \rightarrow \mathbb{R}^{2}$ is a $C^{1}$ function having linear growth, with $f(0)=0$ and $D f(0)$ diagonal, and $g=\left(g_{1}, g_{2}\right): \mathbb{R}^{2} \rightarrow \mathcal{L}\left(\mathbb{R}^{2}\right)$ is any Lipschitz continuous function such that $g(0)$ either vanishes or is diagonal invertible and such that

$$
\|g(\sigma)\|_{\mathcal{L}\left(\mathbb{R}^{2}\right)} \leqslant c\left(1+|\sigma|^{\gamma}\right), \quad \sigma \in \mathbb{R}^{2},
$$

with

$$
2 k+1>(1+6 \gamma) \vee 2 .
$$

In particular, if $g$ is bounded in the reaction term we can take any power $2 k+1 \geqslant 3$.

In [2] it is proved that for any $\varepsilon>0$ and $p \geqslant 1$ system (1.1) admits a unique global solution $u_{\varepsilon}^{x} \in$ $L^{p}(\Omega ; C([0, T] ; E))$, where $E$ is the space of continuous functions on $\overline{\mathcal{O}}$ with values in $\mathbb{R}^{r}$, and for each initial datum $x \in E$ and $a>0$ the family of probability measures $\left\{\mathcal{L}\left(u_{\varepsilon}^{x}(t)\right\}_{t \geqslant a}\right.$ is tight in $(E, \mathcal{B}(E))$. In particular, 
due to the Krylov-Bogoliubov theorem this yields the existence of a sequence $\left\{t_{n}\right\} \uparrow+\infty$ (possibly depending on $\varepsilon$ ) such that the sequence of probability measures defined by

$$
v_{\varepsilon, n}(\Gamma):=\frac{1}{t_{n}} \int_{0}^{t_{n}} \mathbb{P}\left(u_{\varepsilon}^{0}(s) \in \Gamma\right) d s, \quad \Gamma \in \mathcal{B}(E),
$$

converges weakly to some measure $v_{\varepsilon}$, which is invariant for system (1.1).

In the earlier paper [3] we have proved that the process $\left\{u_{\varepsilon}^{x}\right\}_{\varepsilon>0}$ is governed by a large deviation principle in $C([0, T] ; E)$, for any $T>0$. Our aim here is to prove that the family of invariant measures $\left\{v_{\varepsilon}\right\}_{\varepsilon>0}$ defined as the weak limits of the sequences of measures as in (1.3) obeys a large deviation principle in $E$, as $\varepsilon$ goes to zero (precise hypotheses on the coefficients are specified in Section 2 below to which the reader is referred to).

Clearly the first step in the proof of large deviations estimates is defining an appropriate action functional $V$ having compact level sets. The hardest part here is not to find $V$ (see (5.1) below for its initial definition and, in particular, [9] and [14]) but to find a good characterization of it, in order to prove that its level sets are compact and, maybe more importantly, to get a better intuition about its meaning. So, we spend a great deal of effort to prove that (as in [8] and [14]), the action functional $V$, also called quasi-potential, has the following form

$$
V(x)=\min \left\{I_{-\infty}(z) ; z \in C((-\infty, 0] ; E), z(0)=x, \lim _{t \rightarrow-\infty}|z(t)|_{E}=0\right\} .
$$

Here $I_{-\infty}(z)$ is the minimum energy required to produce $z$ as a solution of the skeleton equation corresponding to (1.1) for $t \in(-\infty, 0]$, i.e. replace $\partial w / \partial t$ by a deterministic function $\varphi \in L^{2}\left(-\infty, 0 ; L^{2}\left(\mathcal{O}, \mathbb{R}^{r}\right)\right)$ so that the corresponding solution $z(\varphi)$ equals $z$ and the energy $\left(:=|\varphi|_{L^{2}\left(-\infty, 0 ; L^{2}\left(\mathcal{O}, \mathbb{R}^{r}\right)\right)}^{2}\right)$ is minimal (cf. Section 3 and the beginning of Section 5 below).

By compactness the infimum in (1.4) is indeed achieved by some $z_{0}$, which exhibits more regularity in the space variables than just being in $E$ (cf. Lemma 3.5 which in turn is essential for the proof of Proposition 5.4, but also for the proof of Lemma 7.1 which yields upper bounds). We would like to mention at this point that proving (1.4) requires considerable new input, since we consider space dimension $d \geqslant 1$, so the coefficient in front of the noise (in contrast to the one-dimensional case considered in [9] and [14]) can no longer be invertible. In addition truly degenerate multiplicative noise is included in our framework.

Once we have shown that the mapping $V: E \rightarrow[0,+\infty]$ is lower semi-continuous, with compact level sets, we prove that the family of probability measures $\left\{v_{\varepsilon}\right\}_{\varepsilon>0}$ obeys a principle of large deviations with action functional $V$ (cf. $[9,10]$ and $[14]$ for the formulation), i.e.

1. lower bounds (cf. Section 6 below): for any $\delta, \gamma>0$ and $\bar{x} \in E$ there exists $\varepsilon_{0}>0$ such that

$$
\nu_{\varepsilon}\left(\left\{x \in E:|x-\bar{x}|_{E}<\delta\right\}\right) \geqslant \exp \left(-\frac{V(\bar{x})+\gamma}{\varepsilon^{2}}\right), \quad \varepsilon \leqslant \varepsilon_{0}
$$

2. upper bounds (cf. Section 7 below): for any $s, \delta, \gamma>0$ there exists $\varepsilon_{0}>0$ such that

$$
v_{\varepsilon}\left(\left\{x \in E ; \operatorname{dist}_{E}(x, K(s)) \geqslant \delta\right\}\right)<\exp \left(-\frac{s-\gamma}{\varepsilon^{2}}\right), \quad \varepsilon \leqslant \varepsilon_{0},
$$

where $K(s):=\{x \in E: V(x) \leqslant s\}$ is the level set of $V$.

In accordance to the general ideas about the large deviations for the invariant measures $\left\{v_{\varepsilon}\right\}_{\varepsilon>0}$ (as beautifully explained in the introduction of [9]) we have the following interpretation. Due to the definition of $v_{\varepsilon}$ for any set $A \subset E$ the number $v_{\varepsilon}(A)$ is the mean expected time the process $u_{\varepsilon}$ spends in $A$. Moreover, by the large deviation results in [3] points in $K(s)$, for small $s$, are of course more likely to be visited by $u_{\varepsilon}$. So, according to statement 2 above, the mass of $v_{\varepsilon}$ will concentrate as $\varepsilon \rightarrow 0$ at points in $E$ which are minimum points of $V$. In our case

$$
V(x)=0 \Leftrightarrow x=0
$$


(cf. (5.2) below), so $v_{\varepsilon}$ will converge to the Dirac measure at the zero function in $E$, i.e. the only stationary solution of Eq. (1.1) for $\varepsilon=0$.

In the framework considered in the present paper the skeleton equation associated with system (1.1) is not null controllable, as in the case considered by Sowers in [14]. Then the proof of lower bounds turns out to be more complicate than in [14]. In fact, a crucial role is played by Lemma 6.2, whose proof is not immediate, as we are dealing with non-Lipschitz reaction term, unbounded $G$ and any space dimension $d \geqslant 1$. To this purpose we note that for the proof of Lemma 6.2 we also benefited from some ideas of I. Daw (see [6]).

Concerning the upper bounds, we have distinguished the case of bounded and unbounded $G$. When $G$ is bounded we can use exponential estimates for the solution $u_{\varepsilon}$ proved in [3] and generalize some arguments of Sowers to our more delicate situation. In the case of unbounded $G$ this is not anymore possible. Hence we need to prove estimate (3.13) in Theorem 3.4 below, i.e. an estimate on the solution of the skeleton equation which is uniform with respect to the initial datum. This allows us to prove Theorem 7.5, where $g$ only satisfies the growth condition in Hypothesis 6, without using the exponential tail estimates (7.2) for the solution of (1.1) which are only known to hold for bounded $g$. Thus, Theorem 3.4 turns into a key step, since here we have not succeeded in applying a localization argument as we did in [3].

Finally, let us mention that our general strategy mainly follows R. Sowers [14], but our more general situation requires various new techniques. These, in particular, becomes necessary because of the following.

1. Unlike in [13] and [14] (see also [6] and [11]), where global Lipschitz assumptions were imposed, here the functions $f_{i}$ in (1.1) are only locally Lipschitz and of polynomial growth (see Hypothesis 3 and Remark 2.4 below).

2. $g=\left[g_{i j}\right]$ in (1.1) is not assumed to be globally bounded (as e.g. done in [13] and [6]) and just assumed to be globally Lipschitz (see Hypothesis 2, but also Hypothesis 6 for the proof of upper bounds). Moreover, $g$ may be degenerate. This means that we can consider for example $g_{i j}(u)=\lambda_{i j} u_{j}$, with $\lambda_{i j} \in \mathbb{R}$.

3. We consider systems of $r$ coupled stochastic reaction-diffusion equations, ruling out the maximum principle and hence comparison techniques commonly used in case $r=1$.

4. Unlike in [14], where space dimension $d=1$ is considered, we can allow arbitrary space dimension, i.e. for $E=C\left(\overline{\mathcal{O}} ; \mathbb{R}^{r}\right)$ we can allow $\mathcal{O}$ to be a bounded open subset of $\mathbb{R}^{d}$, for arbitrary $d \geqslant 1$ (cf. Hypothesis 2 below).

\section{Assumptions and preliminaries}

Let $\mathcal{O}$ be a bounded open set of $\mathbb{R}^{d}$, with $d \geqslant 1$, having a $C^{\infty}$ boundary. In what follows we shall denote by $H$ the Hilbert space $L^{2}\left(\mathcal{O} ; \mathbb{R}^{r}\right), r \geqslant 1$, endowed with the usual scalar product $\langle\cdot, \cdot\rangle_{H}$ and the corresponding norm $|\cdot|_{H}$. The norm in $L^{p}\left(\mathcal{O} ; \mathbb{R}^{r}\right), p \in[1, \infty], p \neq 2$, shall be denoted by $|\cdot|_{p}$.

For any $1 \leqslant p \leqslant \infty$ and $m \in \mathbb{N}$, by $W^{m, p}(\mathcal{O})$ we shall denote the space of functions $f \in L^{p}(\mathcal{O})$ such that the weak derivatives $D^{\alpha} f$ exist in $L^{p}(\mathcal{O})$, for each $0 \leqslant|\alpha| \leqslant m$. $W^{m, p}(\mathcal{O})$ is a Banach space, endowed with the norm

$$
|f|_{W^{m, p}(\mathcal{O})}:=\sum_{|\alpha| \leqslant m}\left|D^{\alpha} f\right|_{L^{p}(\mathcal{O})}
$$

Moreover, if $s>0$ is not integer, we define $W^{s, p}(\mathcal{O})$ as the space of functions $f \in W^{[s], p}(\mathcal{O})$ such that

$$
|f|_{W^{s, p}(\mathcal{O})}:=|f|_{W^{[s], p}(\mathcal{O})}+\sum_{|\alpha|=[s]} \int_{\mathcal{O} \times \mathcal{O}} \frac{\left|D^{\alpha} f(\xi)-D^{\alpha} f(\eta)\right|^{p}}{|\xi-\eta|^{d+(s-[s]) p}} d \xi d \eta<\infty .
$$

Next, we recall that for any $s \in \mathbb{R}$ and $p \in(1, \infty)$ the Bessel potential space $H^{s, p}\left(\mathbb{R}^{d}\right)$ is defined by

$$
H^{s, p}\left(\mathbb{R}^{d}\right):=\left\{f \in \mathcal{S}^{\prime}\left(\mathbb{R}^{d}\right):|f|_{H^{s, p}\left(\mathbb{R}^{d}\right)}:=\left|\mathcal{F}^{-1}\left(1+|\xi|^{2}\right)^{s / 2} \mathcal{F} f\right|_{L^{p}\left(\mathbb{R}^{d}\right)}<\infty\right\},
$$


where $\mathcal{S}^{\prime}\left(\mathbb{R}^{d}\right)$ is the space of tempered distributions on $\mathbb{R}^{d}$ and $\mathcal{F}$ the Fourier transform. The Bessel potential spaces on $\mathcal{O}$ are defined by restriction as

$$
H^{s, p}(\mathcal{O}):=\left\{f=g_{\left.\right|_{\partial \mathcal{O}}} ; g \in H^{s, p}\left(\mathbb{R}^{d}\right)\right\},
$$

with

$$
|f|_{H^{s, p}(\mathcal{O})}:=\inf _{f=g_{\left.\right|_{\partial \mathcal{O}}}}|g|_{H^{s, p}\left(\mathbb{R}^{d}\right)} .
$$

We note that for $k \in \mathbb{N}$ we have $H^{k, p}(\mathcal{O})=W^{k, p}(\mathcal{O})$ (for all definitions and detailed proofs see [15]).

Finally, we shall denote by $W^{s, p}\left(\mathcal{O} ; \mathbb{R}^{r}\right)$ and $H^{s, p}\left(\mathcal{O} ; \mathbb{R}^{r}\right)$ the space of $\mathbb{R}^{r}$-valued functions such that each component belongs to $W^{s, p}(\mathcal{O})$ and $H^{s, p}(\mathcal{O})$, respectively.

In what follows we shall denote by $A$ the realization in $H$ of the differential operator $\mathcal{A}=\left(\mathcal{A}_{1}, \ldots, \mathcal{A}_{r}\right)$ defined in (1.2), endowed with the boundary conditions $\mathcal{B}=\left(\mathcal{B}^{1}, \ldots, \mathcal{B}^{r}\right)$, where for each $i=1, \ldots, r$

$$
\mathcal{B}^{i} u=u, \quad \text { or } \quad \mathcal{B}^{i} u=\left\langle a^{i} v, \nabla u\right\rangle,
$$

(here $v$ is the normal vector at $\partial \mathcal{O}$ ). As proved e.g. in [15, Chapter 5] we have

$$
D(A)=\left\{u \in H^{2,2}\left(\mathcal{O} ; \mathbb{R}^{r}\right): \mathcal{B} u=0 \text { on } \partial \mathcal{O}\right\}=: H_{\mathcal{B}}^{2,2}\left(\mathcal{O} ; \mathbb{R}^{r}\right)
$$

and the following optimal regularity result holds

$$
u \in D(A), A u \in H^{l, 2}\left(\mathcal{O} ; \mathbb{R}^{r}\right), l \in \mathbb{N}^{+} \Rightarrow u \in H^{l+2,2}\left(\mathcal{O} ; \mathbb{R}^{r}\right) .
$$

We recall that for any integer $k \geqslant 2$ the $k$ th power of the operator $A$ is defined by

$$
D\left(A^{k}\right):=\left\{u \in D\left(A^{k-1}\right): A^{k-1} u \in D(A)\right\}, \quad A^{k} u:=A\left(A^{k-1} u\right) .
$$

Analogously, we can define the $k$ th power of $\mathcal{A}$ by setting

$$
\mathcal{A}^{k} u:=\mathcal{A}\left(\mathcal{A}^{k-1} u\right)=\left(\mathcal{A}_{1}\left(\mathcal{A}_{1}^{k-1} u_{1}\right), \ldots, \mathcal{A}_{r}\left(\mathcal{A}_{r}^{k-1} u_{r}\right)\right), \quad u \in H^{2 k, 2}\left(\mathcal{O} ; \mathbb{R}^{r}\right) .
$$

Thanks to (2.2) it is immediate to show that for any fixed integer $k$

$$
D\left(A^{k}\right)=H_{\mathcal{B}_{k}}^{2 k, 2}\left(\mathcal{O} ; \mathbb{R}^{r}\right):=\left\{u \in H^{2 k, 2}\left(\mathcal{O} ; \mathbb{R}^{r}\right): \mathcal{B} u=\cdots=\mathcal{B}\left(\mathcal{A}^{k-1} u\right)=0\right\},
$$

so that the operator $A^{k}$ is the realization in $H$ of the differential operator $\mathcal{A}^{k}$ endowed with the boundary conditions

$$
\mathcal{B}_{k}:=\left\{\mathcal{B}, \mathcal{B A}, \ldots, \mathcal{B A}^{k-1}\right\} .
$$

Notice that $A$ generates an analytic semigroup $e^{t A}$ in each $L^{p}\left(\mathcal{O} ; \mathbb{R}^{r}\right)$, with $1 \leqslant p \leqslant \infty$, which is self-adjoint on $H$ and of negative type. Thus, as $-A$ is a positive self-adjoint operator on $H$, for any $0 \leqslant \alpha \leqslant \beta$ and $\theta \in[0,1]$ we have

$$
\left[D\left((-A)^{\alpha}\right), D\left((-A)^{\beta}\right)\right]_{\theta}=\left(D\left((-A)^{\alpha}\right), D\left((-A)^{\beta}\right)\right)_{\theta, 2}=D\left((-A)^{(1-\theta) \alpha+\theta \beta}\right),
$$

where in general, given any two Banach spaces $X$ and $Y,[X, Y]_{\theta}$ denotes their complex interpolation space and $(X, Y)_{\theta, 2}$ denotes their real interpolation space (for a proof see [15, Theorem 1.18.10]).

By complex interpolation arguments it is possible to characterize the domain of the fractional powers of $-A$.

Proposition 2.1. Let $_{i}:=\left(1+2\right.$ ord $\left.\mathcal{B}^{i}\right) / 4$. Then, for any $\gamma>0$ and $i=1, \ldots, r$ we have

$$
D\left((-A)^{\gamma}\right)=H_{\mathcal{B}_{\gamma}}^{2 \gamma, 2}\left(\mathcal{O} ; \mathbb{R}^{r}\right)
$$

where $\mathcal{B}_{\gamma}:=\left(\mathcal{B}_{\gamma}^{1}, \ldots, \mathcal{B}_{\gamma}^{r}\right)$ with

$$
\mathcal{B}_{\gamma}^{i}:= \begin{cases}\emptyset & \text { if } \gamma \in\left[0, m_{i}\right], \\ \left\{\mathcal{B}^{i}, \mathcal{B}^{i} \mathcal{A}_{i}, \ldots, \mathcal{B}^{i} \mathcal{A}_{i}^{k}\right\} & \text { if } \gamma \in\left(k+m_{i}, k+1+m_{i}\right], k \in \mathbb{N} \cup\{0\} .\end{cases}
$$


Proof. Due to (2.4) we have

$$
D\left((-A)^{\gamma}\right)=\left[H, D\left((-A)^{[\gamma]+1}\right)\right]_{\frac{\gamma}{[\gamma]+1}}
$$

and then from (2.3) we obtain

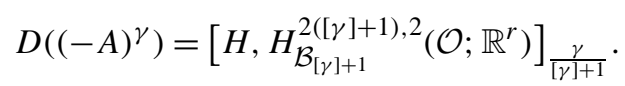

It is not difficult to prove that for any integer $k$ the operator $\mathcal{A}^{k}$ endowed with the boundary condition $\mathcal{B}_{k}$ is regular elliptic (for the definition and all details see [15, Section 5.2.1]). Thus, as proved in [4, Lemma 11] from (2.6) we obtain

$$
D\left((-A)^{\gamma}\right)=H_{\mathcal{B}_{\gamma}}^{2 \gamma, 2}\left(\mathcal{O} ; \mathbb{R}^{r}\right),
$$

where

$$
\mathcal{B}_{\gamma}^{i}:=\left\{\mathcal{B}^{i} \mathcal{A}_{i}^{j} ; 0 \leqslant j \leqslant[\gamma], \operatorname{ord}\left(\mathcal{B}^{i} \mathcal{A}_{i}^{j}\right)<2 \gamma-\frac{1}{2}\right\} .
$$

Hence, by easy computations we can check that the boundary conditions $\mathcal{B}_{\gamma}^{i}$ above coincide with the boundary conditions $\mathcal{B}_{\gamma}^{i}$ in (2.5).

Remark 2.2. It is immediate to check that if $\gamma \in \mathbb{N}$ the boundary conditions $\mathcal{B}_{\gamma}$ introduced in (2.3) coincide with the boundary conditions $\mathcal{B}_{\gamma}$ introduced in the proposition above.

In what follows we shall set

$$
E:=\overline{D(A)} C\left(\overline{\mathcal{O}} ; \mathbb{R}^{r}\right)=\overline{D\left(A_{1}\right)} C(\overline{\mathcal{O}} ; \mathbb{R}) \times \cdots \times \overline{D\left(A_{r}\right)} C(\overline{\mathcal{O}} ; \mathbb{R}) .
$$

Each set $\overline{D\left(A_{i}\right)} C(\overline{\mathcal{O}} ; \mathbb{R})$ coincides with $C(\overline{\mathcal{O}} ; \mathbb{R})$ or $C_{0}(\overline{\mathcal{O}} ; \mathbb{R})$, if $\mathcal{B}_{i}$ is respectively a co-normal or a Dirichlet boundary condition. In any case, with this definition of the space $E$, endowed with the sup-norm $|\cdot|_{E}$ and the duality $\langle\cdot, \cdot\rangle_{E}:=_{E^{\star}}\langle\cdot, \cdot\rangle_{E}$, the part of $e^{t A}$ in $E$ (which we will still denote by $e^{t A}$ ) is strongly continuous. Moreover for any $\delta_{x} \in \partial|x|_{E}:=\left\{x^{\star} \in E^{\star},\left\langle x, x^{\star}\right\rangle_{E}=|x|_{E},\left|x^{\star}\right|_{E^{\star}}=1\right\}$ we have

$$
\left\langle A x, \delta_{x}\right\rangle_{E} \leqslant-\alpha|x|_{E}, \quad x \in D(A),
$$

where $\alpha:=\min _{i=1, \ldots, r} \alpha_{i}$.

As recalled also in [2] and [3], $e^{t A}$ has a smoothing effect. In fact, for any $t>0,1 \leqslant q \leqslant p \leqslant \infty$ and $\varepsilon \geqslant 0$ the semigroup $e^{t A}$ maps $L^{q}\left(\mathcal{O} ; \mathbb{R}^{r}\right)$ into $W^{\varepsilon, p}\left(\mathcal{O} ; \mathbb{R}^{r}\right)$ and

$$
\left|e^{t A} x\right|_{W^{\varepsilon, p}\left(\mathcal{O} ; \mathbb{R}^{r}\right)} \leqslant c e^{-\alpha t}(t \wedge 1)^{-\left(\frac{\varepsilon}{2}+\frac{d(p-q)}{2 p q}\right)}|x|_{q}, \quad x \in L^{q}\left(\mathcal{O} ; \mathbb{R}^{r}\right) .
$$

Moreover, $e^{t A}$ maps $E$ into $C^{\theta}\left(\overline{\mathcal{O}} ; \mathbb{R}^{r}\right)$, for any $\theta \geqslant 0$, and

$$
\left|e^{t A} x\right|_{C^{\theta}\left(\overline{\mathcal{O}} ; \mathbb{R}^{r}\right)} \leqslant c e^{-\alpha t}(t \wedge 1)^{-\frac{\theta}{2}}|x|_{E}, \quad x \in E .
$$

We also notice that $e^{t A}$ is compact on $L^{p}\left(\mathcal{O} ; \mathbb{R}^{r}\right)$, for all $1 \leqslant p \leqslant \infty$ and $t>0$, and the spectrum $\left\{-\alpha_{n}\right\}$ is independent of $p$.

Our first hypothesis concerns the eigenvalues of $A$.

Hypothesis 1. The complete orthonormal system of $H$ which diagonalizes A is equi-bounded in the sup-norm.

Next, we assume that $Q:=\left(Q_{1}, \ldots, Q_{r}\right): H \rightarrow H$ is a bounded linear operator which satisfies the following conditions. 
Hypothesis 2. $Q$ is non-negative and diagonal with respect to the complete orthonormal basis which diagonalizes $A$, with eigenvalues $\left\{\lambda_{n}\right\}$. Moreover, if $d \geqslant 2$,

$$
\text { there exists }\left\{\begin{array}{ll}
\varrho<\infty & \text { if } d=2 \\
\varrho<\frac{2 d}{d-2} & \text { if } d>2
\end{array} \text { such that }\|Q\|_{\varrho}:=\left(\sum_{k=1}^{\infty} \lambda_{n}^{\varrho}\right)^{1 / \varrho}<\infty .\right.
$$

Remark 2.3. Hypothesis 1 is satisfied e.g. by the Laplace operator on $[0, T]^{d}$ endowed with Dirichlet boundary conditions. But there are several important cases in which it is not satisfied and it is only possible to say that

$$
\left|e_{k}\right|_{\infty} \leqslant c k^{\gamma}
$$

for some $\gamma \geqslant 0$. In this more general situation one has to assume that the summability condition (2.10) imposed on the eigenvalues of $Q$ is satisfied for some smaller constant $\varrho^{\prime}$. In other words one has to color the noise more.

In Hypotheses 3 and 4 below we give conditions on the coefficients $f$ and $g$.

Hypothesis 3. The mapping $g: \overline{\mathcal{O}} \times \mathbb{R}^{r} \rightarrow \mathcal{L}\left(\mathbb{R}^{r}\right)$ is continuous. Moreover the mapping $g(\xi, \cdot): \mathbb{R}^{r} \rightarrow \mathcal{L}\left(\mathbb{R}^{r}\right)$ is Lipschitz-continuous, uniformly with respect to $\xi \in \overline{\mathcal{O}}$, that is

$$
\sup _{\xi \in \overline{\mathcal{O}}} \sup _{\substack{\sigma, \rho \in \mathbb{R}^{r} \\ \sigma \neq \rho}} \frac{\|g(\xi, \sigma)-g(\xi, \rho)\|_{\mathcal{L}\left(\mathbb{R}^{r}\right)}}{|\sigma-\rho|}<\infty
$$

In what follows for any $x, y: \overline{\mathcal{O}} \rightarrow \mathbb{R}^{r}$ we set

$$
(G(x) y)(\xi):=g(\xi, x(\xi)) y(\xi), \quad \xi \in \overline{\mathcal{O}} .
$$

Next, setting $f:=\left(f_{1}, \ldots, f_{r}\right)$, for any $x: \overline{\mathcal{O}} \rightarrow \mathbb{R}^{r}$ we define

$$
F(x)(\xi):=f(\xi, x(\xi)), \quad \xi \in \overline{\mathcal{O}} .
$$

\section{Hypothesis 4.}

(1) The mapping $F: E \rightarrow E$ is locally Lipschitz-continuous and there exists $m \geqslant 1$ such that

$$
|F(x)|_{E} \leqslant c\left(1+|x|_{E}^{m}\right), \quad x \in E .
$$

Moreover, $F(0)=0$.

(2) For any $x, h \in E$

$$
\left\langle F(x+h)-F(x), \delta_{h}\right\rangle_{E} \leqslant 0,
$$

for some $\delta_{h} \in \partial|h|_{E}:=\left\{h^{\star} \in E^{\star} ;\left|h^{\star}\right|_{E^{\star}}=1,\left\langle h, h^{\star}\right\rangle_{E}=|h|_{E}\right\}$.

(3) There exist $a>0$ and $c \geqslant 0$ such that for each $x, h \in E$

$$
\left\langle F(x+h)-F(x), \delta_{h}\right\rangle_{E} \leqslant-a|h|_{E}^{m}+c\left(1+|x|_{E}^{m}\right),
$$

for some $\delta_{h} \in \partial|h|_{E}$.

Remark 2.4. Assume that

$$
f_{i}\left(\xi, \sigma_{1}, \ldots, \sigma_{r}\right):=k_{i}\left(\xi, \sigma_{i}\right)+h_{i}\left(\xi, \sigma_{1}, \ldots, \sigma_{r}\right), \quad i=1, \ldots, r,
$$


where $h_{i}: \overline{\mathcal{O}} \times \mathbb{R}^{r} \rightarrow \mathbb{R}$ is a continuous function such that $h_{i}(\xi, \cdot): \mathbb{R}^{r} \rightarrow \mathbb{R}$ is locally Lipschitz-continuous with linear growth, uniformly with respect to $\xi \in \overline{\mathcal{O}}$, and

$$
k_{i}\left(\xi, \sigma_{i}\right):=-c(\xi) \sigma_{i}^{2 n+1}+\sum_{k=0}^{2 n} c_{k}(\xi) \sigma_{i}^{k},
$$

where $c(\xi)$ and $c_{k}(\xi)$ are continuous functions, $c(\xi) \geqslant \varepsilon>0, \xi \in \overline{\mathcal{O}}$ and $c_{0}(\xi)=-h_{i}(\xi, 0)$.

Under these assumptions the function $f$ satisfies conditions (1) and (3) in Hypothesis 4 (see also [1, Chapter 6], [2] and [3, Remark 2.1] for more general examples of functions $f$ fulfilling Hypothesis 4 and for all details).

The next set of conditions assure the compactness of level sets for the quasi-potential associated with system (1.1).

Hypothesis 5. Either $G(0)=0$ or there exists a continuous increasing function $c(t)$ such that for any $t \geqslant 0$

$$
\left|Q[G(0)]^{\star} e^{t\left[A+F^{\prime}(0)\right]^{\star}} h\right|_{H} \geqslant c(t)\left|Q e^{t A} h\right|_{H}, \quad h \in H .
$$

In the case (2.14) is verified, the following conditions hold.

(1) If $\left\{-\alpha_{n}\right\}$ and $\left\{\lambda_{n}\right\}$ are respectively the eigenvalues of $A$ and $Q$, then

$$
\frac{1}{c} \alpha_{n}^{-\delta} \leqslant \lambda_{n} \leqslant c \alpha_{n}^{-\delta}
$$

for some $c>0$ and some $\delta$ such that

$$
\delta \geqslant 0, \quad \text { if } d=1, \quad \delta>\frac{d-2}{4}, \quad \text { if } d \geqslant 2 .
$$

(2) The mappings $f$ and $g$ are of class $C^{\infty}$ on $\overline{\mathcal{O}} \times \mathbb{R}^{r}$.

(3) If $\delta$ is the constant in (2.15) and $\mathcal{B}_{\gamma}$ is the boundary operator introduced in (2.5), then for any $\gamma \leqslant \delta$ and $u, v \in H^{2 \gamma, 2}\left(\mathcal{O} ; \mathbb{R}^{r}\right)$ we have

$$
\begin{aligned}
& \mathcal{B}_{\gamma} u_{\left.\right|_{\partial O}}=0 \Rightarrow \mathcal{B}_{\gamma} F(u)_{\left.\right|_{\partial O}}=0, \\
& \mathcal{B}_{\gamma} u_{\left.\right|_{\partial O}}=\mathcal{B}_{\gamma} v_{\left.\right|_{\partial O}}=0 \Rightarrow \mathcal{B}_{\gamma}(G(u) v)_{\left.\right|_{\partial O}}=0 .
\end{aligned}
$$

Moreover, if $u, v, w \in H^{2 \delta, 2}\left(\mathcal{O} ; \mathbb{R}^{r}\right)$ we have

$$
\begin{aligned}
& \mathcal{B}_{\delta} u_{\left.\right|_{\partial O}}=\mathcal{B}_{\delta} v_{\mid \partial O}=0 \Rightarrow \mathcal{B}_{\delta}\left(F^{\prime}(u) v\right)_{\mid \partial O}=0, \\
& \mathcal{B}_{\delta} u_{\left.\right|_{\partial} O}=\mathcal{B}_{\delta} v_{\left.\right|_{\partial O}}=\mathcal{B}_{\delta} w_{\left.\right|_{\partial O}}=0 \Rightarrow \mathcal{B}_{\delta}\left(\left[G^{\prime}(u) v\right] w\right)_{\mid \partial O}=0 .
\end{aligned}
$$

\section{Remark 2.5.}

1. We note that the assumption (2.14) is fulfilled when there exist two diagonal $r \times r$ matrices $D_{1}$ and $D_{2}$, with $D_{1}$ invertible, such that

$$
g(\xi, 0)=D_{1}, \quad D_{\sigma} f(\xi, 0)=D_{2}, \quad \xi \in \overline{\mathcal{O}} .
$$

In particular, when instead of a system a single equation is considered, condition (2.14) is always fulfilled if both $g(\xi, 0)$ and $D_{\sigma} f(\xi, 0)$ do not depend on $\xi$.

2. Condition (2.15) means that Range $Q=D\left((-A)^{\delta}\right)$.

3. We assume $f$ and $g$ to be $C^{\infty}\left(\overline{\mathcal{O}} \times \mathbb{R}^{r}\right)$ only for simplicity. In fact we need $f$ and $g$ to be of class $C^{k}\left(\overline{\mathcal{O}} \times \mathbb{R}^{r}\right)$, for some $k$ large enough, depending on the constant $\delta$ introduced in (2.15) (for example, in the case $\mathcal{B}^{i}=I$ it is sufficient to take $k<2 \delta+1 / 2$, see also next remark). 
4. If we have

$$
\alpha_{n} \sim n^{2 / d}, \quad n \in \mathbb{N}
$$

(this happens for example in the case of the Laplace operator $\Delta$ in strongly regular open sets, both with Dirichlet and with Neumann boundary conditions, see [5, Theorem 1.9.6]), then if (2.16) holds, there exists some $\rho$ which fulfills condition (2.10).

5. When $\mathcal{B}^{i}=I$, for each $i=1, \ldots, r$, condition (2.17) is verified for example by functions $f$ and $g$ such that

$$
\mathcal{D}_{\sigma}^{j} f(\xi, 0)=0, \quad D_{\sigma}^{j} g(\xi, 0)=0, \quad \xi \in \overline{\mathcal{O}},
$$

for any $j=1, \ldots, 2 k$, where $k \in\left[\delta-5 / 4, \delta-1 / 4\right.$ ) (notice that in this case $m_{i}=1 / 4$, for each $i$ ). In the same setting, condition (2.18) holds for $f$ and $g$ fulfilling (2.19) for any $j=1, \ldots, 2 k+1$, with $k$ as above.

For the proof of upper bounds in the case of unbounded $g$ we need the following condition on its growth.

Hypothesis 6. There exists $\gamma \in[0,1]$ such that

$$
\sup _{\xi \in \overline{\mathcal{O}}}|g(\xi, \sigma)|_{\mathcal{L}\left(\mathbb{R}^{r}\right)} \leqslant c\left(1+|\sigma|^{\gamma}\right), \quad \sigma \in \mathbb{R}^{r}
$$

and

$$
m>\left[1+(2+d) \gamma\left(1-\frac{d(\varrho-2)}{2 \varrho}\right)^{-1}\right] \vee 2,
$$

where $\varrho$ and $m$ are the constants introduced respectively in (2.10) and (2.13).

Remark 2.6. Condition (2.21) on $d, m, \varrho$ and $\gamma$ says how the space dimension, the dissipativity of $F$, the regularity of $Q$ and the growth of $G$ are related to one another, in order to have upper bounds.

In the case of space dimension $d=1$ and white noise (which means $Q=I$ and hence $\varrho=+\infty$ ) the relation between $m$ (the dissipativity of $F$ ) and $\gamma$ (the growth of $G$ ) is

$$
m>(1+6 \gamma) \vee 2,
$$

so that in the case of $G$ having linear growth (that is $\gamma=1$ ) we have to assume $m>7$. If instead of a white noise we take a coloured noise with Hilbert-Schmidt covariance $Q$ (that is $\varrho=2$ ) we have

$$
m>(1+3 \gamma) \vee 2
$$

which becomes $m>4$ in the case of $\gamma=1$.

In general, from (2.10) we have that the bigger the space dimension $d$ becomes, the smaller $\varrho$ has to be chosen (and hence the more regular $Q$ has to be taken). Due to (2.21) this means that if we want to allow the same growth of $g$ with increasing dimensions, we have to take reaction terms $F$ with stronger and stronger dissipativity, that is, larger and larger $m$.

\section{The skeleton equation}

With the notations introduced in the previous section system (1.1) can written more concisely as

$$
d u(t)=[A u(t)+F(u(t))] d t+G(u(t)) Q d w(t), \quad u(0)=x .
$$

In this section we prove some results for the skeleton equation associated with the system above. 
For any $-\infty \leqslant t_{1}<t_{2} \leqslant+\infty$ and $\varphi \in L^{2}\left(t_{1}, t_{2} ; H\right)$ we denote by $z(\varphi)$ any solution belonging to $C\left(\left[t_{1}, t_{2}\right] ; E\right)$ of the deterministic problem

$$
z^{\prime}(t)=A z(t)+F(z(t))+G(z(t)) Q \varphi(t), \quad z\left(t_{1}\right)=x .
$$

In several cases, when we need to stress that $z(\varphi)$ starts from $x$ at time $t_{1}$, we shall write $z_{t_{1}}^{x}(\varphi)$. As shown in [3, Theorem 4.1], for any $r \geqslant 0$ and $t_{1}<t_{2}$ there exists a constant $c_{r, t_{2}-t_{1}}>0$ such that for any $x \in E$

$$
\sup _{|\varphi|_{L^{2}\left(t_{1}, t_{2} ; H\right)} \leqslant r}\left|z^{x}(\varphi)\right|_{C\left(\left[t_{1}, t_{2}\right] ; E\right)} \leqslant c_{r, t_{2}-t_{1}}\left(1+|x|_{E}\right) .
$$

In fact, by proceeding as in [2, proofs of Proposition 6.1 and Theorem 6.2], it is possible to get the following stronger result.

Theorem 3.1. Under Hypotheses $1-4$, for any $r \geqslant 0$ there exists a constant $c_{r}>0$ such that for any $T \in \mathbb{R}$ and $x \in E$

$$
\sup _{|\varphi|_{L^{2}(T, \infty ; H)} \leqslant r}\left|z_{T}^{x}(\varphi)\right|_{C([T, \infty) ; E)} \leqslant c_{r}\left(1+|x|_{E}\right) .
$$

Moreover, there exists $\theta_{\star} \in(0,1)$ and $c_{r} \in(0,+\infty)$ such that for any $t>T$ and $x \in E$

$$
\sup _{|\varphi|_{L^{2}(T, \infty ; H)} \leqslant r}\left|z_{T}^{x}(\varphi)(t)\right|_{C^{\theta_{*}}\left(\overline{\mathcal{O}} ; \mathbb{R}^{r}\right)} \leqslant c_{r}\left(1+|x|_{E}^{m}\right)\left(1+(t-T)^{-\frac{\theta_{*}}{2}}\right) .
$$

Proof. For any fixed $\varphi \in L^{2}(T, \infty ; H), z \in C([T, \infty) ; E)$ and $\lambda \geqslant 0$ we define

$$
\gamma_{\varphi, \lambda}^{T}(z)(t):=\int_{T}^{t} e^{(t-s)(A-\lambda)} G(z(s)) Q \varphi(s) d s, \quad t \geqslant T,
$$

(and we set $\gamma_{\varphi}^{T}(z):=\gamma_{\varphi, 0}^{T}(z)$ ). Clearly, $\gamma_{\varphi, \lambda}^{T}(z)$ is the unique mild solution of the problem

$$
\frac{d v}{d t}(t)=(A-\lambda) v(t)+G(z(t)) Q \varphi(t), \quad t \geqslant T, \quad v(T)=0 .
$$

Thanks to the same arguments used in [2, proofs of Theorem 4.2 and Proposition 4.5, Remark 4.6], due to (2.9) we can fix some $\theta_{\star} \in(0,1)$ such that for any $\lambda \geqslant 0$ and $T \in \mathbb{R}$

$$
\sup _{t \geqslant T}\left|\gamma_{\varphi, \lambda}^{T}(z)(t)\right|_{C_{\star}^{\theta_{\star}}\left(\overline{\mathcal{O}} ; \mathbb{R}^{r}\right)} \leqslant c(\lambda)\left(1+|z|_{C([T, \infty) ; E)}\right)|\varphi|_{L^{2}(T, \infty ; H)},
$$

for a constant $c(\lambda)$ decreasing to zero as $\lambda$ goes to infinity.

Now, if we set $\gamma^{T}(t):=\gamma_{\varphi, \lambda}^{T}\left(z_{T}^{x}(\varphi)\right)(t)$ and $u(t):=z_{T}^{x}(\varphi)(t)-\gamma^{T}(t)$, for $t \geqslant T$, we have

$$
u^{\prime}(t)=(A-\lambda) u(t)+F\left(u(t)+\gamma^{T}(t)\right)+\lambda z_{T}^{x}(\varphi)(t), \quad u(T)=x .
$$

We recall here that if a mapping $u:[0, T] \rightarrow E$ is differentiable at some point $t_{0}$ then

$$
\frac{d}{d t}^{-}\left|u\left(t_{0}\right)\right|_{E}=\min \left\{\left\langle u^{\prime}\left(t_{0}\right), x^{\star}\right\rangle_{E}, x^{\star} \in \partial\left|u\left(t_{0}\right)\right|_{E}\right\}
$$

see for example [1, Proposition A.1.3]. Hence, if $\delta_{u(t)}$ is the element of $\partial|u(t)|_{E}$ introduced in (2.13), due to (2.11) we have

$$
\begin{aligned}
\frac{d}{d t}^{-}|u(t)|_{E} \leqslant & \left\langle A u(t), \delta_{u(t)}\right\rangle_{E}+\left\langle F\left(u(t)+\gamma^{T}(t)\right)-F\left(\gamma^{T}(t)\right), \delta_{u(t)}\right\rangle_{E} \\
& +\left\langle F\left(\gamma^{T}(t)\right)+\lambda z_{T}^{x}(\varphi)(t), \delta_{u(t)}\right\rangle_{E} \leqslant-a|u(t)|_{E}^{m}+c\left(1+\left|\gamma^{T}(t)\right|_{E}^{m}+\lambda\left|z_{T}^{x}(\varphi)(t)\right|_{E}\right) .
\end{aligned}
$$


Then, recalling that $z_{T}^{x}(\varphi)=u+\gamma_{\varphi, \lambda}^{T}\left(z_{T}^{x}(\varphi)\right)$, by a comparison argument (see for example [2, proof of Lemma 5.4]) for any $t \geqslant T$ we obtain

$$
\left|z_{T}^{x}(\varphi)(t)\right|_{E} \leqslant|x|_{E}+c\left(1+\sup _{r \geqslant T}\left|\gamma_{\varphi, \lambda}^{T}\left(z_{T}^{x}(\varphi)\right)(r)\right|_{E}+\lambda^{\frac{1}{m}}\left|z_{T}^{x}(\varphi)(t)\right|_{E}^{\frac{1}{m}}\right) .
$$

Thanks to (3.5) and to the Young inequality, this implies that if $|\varphi|_{L^{2}(T, \infty ; H)} \leqslant r$

$$
\sup _{t \geqslant T}\left|z_{T}^{x}(\varphi)(t)\right|_{E} \leqslant|x|_{E}+\frac{1}{4} \sup _{t \geqslant T}\left|z_{T}^{x}(\varphi)(t)\right|_{E}+c(\lambda)\left(1+\left|z_{T}^{x}(\varphi)\right|_{C([T, \infty) ; E)}\right) r+\lambda^{\frac{1}{m-1}}
$$

Now, as $\lim _{\lambda \rightarrow \infty} c(\lambda)=0$, we can find $\bar{\lambda}$ such that $c(\bar{\lambda}) r \leqslant 1 / 4$ and then

$$
\sup _{t \geqslant T}\left|z_{T}^{x}(\varphi)(t)\right|_{E} \leqslant c_{r}\left(1+|x|_{E}\right)
$$

for some positive constant $c_{r}$.

Finally, in order to obtain (3.4), we remark that thanks to (2.9), (2.11) and (3.3) for any $t \geqslant T$ we easily have

$$
\begin{aligned}
& \left|\int_{T}^{t} e^{(t-s) A} F\left(z_{T}^{x}(\varphi)(s)\right) d s\right|_{C^{\theta_{\star}}\left(\overline{\mathcal{O}} ; \mathbb{R}^{r}\right)} \\
& \quad \leqslant c \int_{T}^{t} e^{-\alpha(t-s)}((t-s) \wedge 1)^{-\frac{\theta_{\star}}{2}}\left(1+\left|z_{T}^{x}(\varphi)(s)\right|_{E}^{m}\right) d s \leqslant c_{r}\left(1+|x|_{E}^{m}\right) .
\end{aligned}
$$

Then, as

$$
z_{T}^{x}(\varphi)(t)=e^{(t-T) A} x+\int_{T}^{t} e^{(t-s) A} F\left(z_{T}^{x}(\varphi)(s)\right) d s+\gamma_{\varphi}^{T}\left(z_{T}^{x}(\varphi)\right)(t),
$$

from (3.3), (3.5) and (2.9) for any $t>T$ we get

$$
\left|z_{T}^{x}(\varphi)(t)\right|_{C^{\theta_{\star}}\left(\overline{\mathcal{O}} ; \mathbb{R}^{r}\right)} \leqslant c\left(e^{-\alpha(t-T)}((t-T) \wedge 1)^{-\frac{\theta_{\star}}{2}}|x|_{E}+c_{r}\left(1+|x|_{E}^{m}\right)\right),
$$

which easily implies (3.4)

The next proposition shows that if we start from $x=0$ at time $T$, then $z_{T}^{0}(\varphi)$ decreases to zero in $C([T, \infty) ; E)$, as $\varphi$ decreases to zero in $L^{2}(T, \infty ; H)$.

Proposition 3.2. Under Hypotheses $1-4$, for any $T \in \mathbb{R}$ we have

$$
\lim _{|\varphi|_{L^{2}(T, \infty ; H)} \rightarrow 0}\left|z_{T}^{0}(\varphi)\right|_{C([T, \infty) ; E)}=0 .
$$

Proof. As in the proof of Theorem 3.1, if we set $u(t):=z_{T}^{0}(\varphi)(t)-\gamma_{\varphi}^{T}\left(z_{T}^{0}(\varphi)\right)(t)$, we have

$$
u^{\prime}(t)=A u(t)+F\left(u(t)+\gamma_{\varphi}^{T}\left(z_{T}^{0}(\varphi)\right)(t)\right), \quad u(T)=0,
$$

so that, with the notations of Theorem 3.1,

$$
\begin{aligned}
\frac{d^{-}}{d t}|u(t)|_{E} \leqslant & \left\langle A u(t), \delta_{u(t)}\right\rangle_{E}+\left\langle F\left(u(t)+\gamma_{\varphi}^{T}\left(z_{T}^{0}(\varphi)\right)(t)\right)-F\left(\gamma_{\varphi}^{T}\left(z_{T}^{0}(\varphi)\right)(t)\right), \delta_{u(t)}\right\rangle_{E} \\
& +\left\langle F\left(\gamma_{\varphi}^{T}\left(z_{T}^{0}(\varphi)\right)(t)\right), \delta_{u(t)}\right\rangle_{E} \leqslant-a|u(t)|_{E}^{m}+\left|F\left(\gamma_{\varphi}^{T}\left(z_{T}^{0}(\varphi)\right)(t)\right)\right|_{E} .
\end{aligned}
$$


Recalling that $u(t):=z_{T}^{0}(\varphi)(t)-\gamma_{\varphi}^{T}\left(z_{T}^{0}(\varphi)\right)(t)$, by comparison this yields

$$
\begin{aligned}
\sup _{t \geqslant T}\left|z_{T}^{0}(\varphi)(t)\right|_{E} & \leqslant \sup _{t \geqslant T}\left(|u(t)|_{E}+\left|\gamma_{\varphi}^{T}\left(z_{T}^{0}(\varphi)\right)(t)\right|_{E}\right) \\
& \leqslant c \sup _{t \geqslant T}\left(\left|\gamma_{\varphi}^{T}\left(z_{T}^{0}(\varphi)\right)(t)\right|_{E}+\left.\left|F\left(\gamma_{\varphi}^{T}\left(z_{T}^{0}(\varphi)\right)(t)\right)\right|_{E}\right|^{\frac{1}{m}}\right) .
\end{aligned}
$$

Now, thanks to (3.5) and (3.3), if $|\varphi|_{L^{2}(T, \infty ; H)} \leqslant r$ we have

$$
\sup _{t \geqslant T}\left|\gamma_{\varphi}^{T}\left(z_{T}^{0}(\varphi)\right)(t)\right|_{E} \leqslant c_{r}|\varphi|_{L^{2}(T, \infty ; H)},
$$

and then, as $F(0)=0$, we can conclude.

Now we show that under the growth conditions of Hypothesis 6 it is possible to give estimates of $\left|z^{x}(\varphi)(t)\right|_{E}$ which are uniform with respect to the initial datum $x$. To this purpose we need a preliminary result on the convolution $\gamma_{\varphi}^{0}(z)$.

Lemma 3.3. Let us assume Hypotheses 1-4 and 6. Then, if $\varrho$ and $\gamma$ are the constants introduced in (2.10) and (2.20), respectively, for any $q \geqslant 1$ such that

$$
\frac{q}{\gamma} \geqslant 1, \quad \frac{(2+d) \gamma}{q}<1-\frac{d(\varrho-2)}{2 \varrho}
$$

there exists some continuous increasing function $c_{q}(t)$ vanishing at $t=0$ such that for any $z \in L^{q}(0,+\infty ; E)$ and $\varphi \in L^{2}(0,+\infty ; H)$

$$
\left|\gamma_{\varphi}^{0}(z)(t)\right|_{E} \leqslant c_{q}(t)\left(1+|z|_{L^{q}(0, t ; E)}^{\gamma}\right)|\varphi|_{L^{2}(0, t ; H)}, \quad t \geqslant 0 .
$$

Proof. For any $\beta \in(0,1)$ and $t \geqslant 0$ we have

$$
\gamma_{\varphi}^{0}(z)(t)=\frac{\sin \pi \beta}{\pi} \int_{0}^{t}(t-s)^{\beta-1} e^{(t-s) A} v_{\beta}(s) d s,
$$

where

$$
v_{\beta}(s):=\int_{0}^{s}(s-\sigma)^{-\beta} e^{(s-\sigma) A} G(z(\sigma)) Q \varphi(\sigma) d \sigma .
$$

Thanks to (2.8), for any $\beta \in(0,1), \varepsilon>0$ and $p \geqslant 1$ such that $(\beta-1-\varepsilon / 2) p /(p-1)>-1$ we have

$$
\begin{aligned}
\left|\gamma_{\varphi}^{0}(z)(t)\right|_{W^{\varepsilon, p}\left(\mathcal{O} ; \mathbb{R}^{r}\right)} & \leqslant \frac{\sin \pi \beta}{\pi} \int_{0}^{t}(t-s)^{\beta-1-\frac{\varepsilon}{2}}\left|v_{\beta}(s)\right|_{p} d s \\
& \leqslant \frac{\sin \pi \beta}{\pi}\left(\int_{0}^{t}\left|v_{\beta}(s)\right|_{p}^{p} d s\right)^{\frac{1}{p}}\left(\int_{0}^{t}(t-s)^{\left(\beta-1-\frac{\varepsilon}{2}\right) \frac{p}{p-1}} d s\right)^{\frac{p-1}{p}} .
\end{aligned}
$$

Hence, if $\varepsilon p>d$, that is, if

$$
\beta>(2+d) / 2 p
$$


we get

$$
\left|\gamma_{\varphi}^{0}(z)(t)\right|_{E} \leqslant c_{p}(t)\left|v_{\beta}\right|_{L^{p}\left((0, t) \times \mathcal{O} ; \mathbb{R}^{r}\right)}
$$

for some continuous increasing function $c_{p}(t)$ vanishing at $t=0$. Now, for $(s, \xi) \in[0, T] \times \mathcal{O}$ we have

$$
\begin{aligned}
v_{\beta}(s, \xi) & =\int_{0}^{s}(s-\sigma)^{-\beta} \sum_{k=1}^{\infty} e^{(s-\sigma) A}\left[G(z(\sigma)) Q e_{k}\right](\xi)\left\langle\varphi(\sigma), e_{k}\right\rangle_{H} d \sigma \\
& =\int_{0}^{s}(s-\sigma)^{-\beta} \sum_{k=1}^{\infty} \lambda_{k} e^{(s-\sigma) A}\left[G(z(\sigma)) e_{k}\right](\xi)\left\langle\varphi(\sigma), e_{k}\right\rangle_{H} d \sigma
\end{aligned}
$$

and then

$$
\begin{aligned}
\left|v_{\beta}(s, \xi)\right| & \leqslant \int_{0}^{s}(s-\sigma)^{-\beta}\left(\sum_{k=1}^{\infty}\left|\left\langle\varphi(\sigma), e_{k}\right\rangle_{H}\right|^{2}\right)^{\frac{1}{2}}\left(\sum_{k=1}^{\infty} \lambda_{k}^{2}\left|e^{(s-\sigma) A}\left[G(z(\sigma)) e_{k}\right](\xi)\right|^{2}\right)^{\frac{1}{2}} d \sigma \\
& \leqslant\left(\int_{0}^{s}|\varphi(\sigma)|_{H}^{2} d \sigma\right)^{\frac{1}{2}}\left(\int_{0}^{s}(s-\sigma)^{-2 \beta} \sum_{k=1}^{\infty} \lambda_{k}^{2}\left|e^{(s-\sigma) A}\left[G(z(\sigma)) e_{k}\right](\xi)\right|^{2} d \sigma\right)^{\frac{1}{2}} \\
& \leqslant\|Q\|_{\varrho}|\varphi|_{L^{2}(0, s ; H)}\left(\int_{0}^{s}(s-\sigma)^{-2 \beta}\left(\sum_{k=1}^{\infty}\left|e^{(s-\sigma) A}\left[G(z(\sigma)) e_{k}\right](\xi)\right|^{2 \zeta}\right)^{\frac{1}{5}} d \sigma\right)^{\frac{1}{2}}
\end{aligned}
$$

where $\varsigma=\varrho /(\varrho-2)$ and $\varrho=+\infty$ if $d=1$, or $\varrho<2 d /(d-2)$, if $d \geqslant 2$ (see (2.10) in Hypothesis 4).

Now, as shown in [2, Proof of Theorem 4.2], we have

$$
\sum_{k=1}^{\infty}\left|e^{(s-\sigma) A}\left[G(z(\sigma)) e_{k}\right](\xi)\right|^{2 \varsigma} \leqslant c(s-\sigma)^{-\frac{d}{2}}\left|e^{(s-\sigma) A} \zeta(\cdot, z(\sigma))\right|_{E}^{2(\varsigma-1)}\left|e^{(s-\sigma) A} \bar{\zeta}(\cdot, z(\sigma))\right|_{E},
$$

where the functions $\zeta, \bar{\zeta}: \overline{\mathcal{O}} \times \mathbb{R}^{r} \rightarrow \mathbb{R}^{r}$ are defined by

$$
\zeta_{i}(\xi, \rho):=\sum_{j=1}^{r}\left|g_{i j}(\xi, \rho)\right|, \quad \bar{\zeta}_{i}(\xi, \rho):=\sum_{j=1}^{r}\left|g_{i j}(\xi, \rho)\right|^{2}, \quad i=1, \ldots, r .
$$

According to (2.20) this yields

$$
\sum_{k=1}^{\infty}\left|e^{(s-\sigma) A}\left[G(z(\sigma)) e_{k}\right](\xi)\right|^{2 \varsigma} \leqslant c(s-\sigma)^{-\frac{d}{2}}\left(1+|z(\sigma)|_{E}^{2 \gamma \zeta}\right), \quad \xi \in \overline{\mathcal{O}},
$$

and then, if

$$
2 \beta+\frac{d}{2 \varsigma}=2 \beta+\frac{d(\varrho-2)}{2 \varrho}<1,
$$

collecting all terms, from the Young inequality we get

$$
\begin{aligned}
\left|v_{\beta}\right|_{L^{p}\left((0, t) \times ; \mathbb{R}^{r}\right)}^{p} & \leqslant c|\varphi|_{L^{2}(0, t ; H)}^{p} \int_{0}^{t}\left(\int_{0}^{s}(s-\sigma)^{-\left(2 \beta+\frac{d}{2 \varsigma}\right)}\left(1+|z(\sigma)|_{E}^{2 \gamma}\right) d \sigma\right)^{\frac{p}{2}} d s \\
& \leqslant c|\varphi|_{L^{2}(0, t ; H)}^{p}\left(\int_{0}^{t} s^{-\left(2 \beta+\frac{d}{2 \varsigma}\right)} d s\right)^{\frac{p}{2}} \int_{0}^{t}\left(1+|z(s)|_{E}^{p \gamma}\right) d s .
\end{aligned}
$$


Hence, as we can take $p=q / \gamma \geqslant 1$, for some $q \geqslant 1$ fulfilling (3.8), it is possible to fix $\beta \in(0,1)$ fulfilling both (3.9) and (3.11) and thanks to (3.10) and (3.12) we obtain our lemma.

Theorem 3.4. Assume that Hypotheses $1-4$ and 6 hold. Then for any $r \geqslant 0$ there exists $c_{r}>0$ such that

$$
\sup _{x \in E} \sup _{|\varphi|_{L^{2}(0, \infty ; H)} \leqslant r}\left|z^{x}(\varphi)(t)\right|_{E} \leqslant c_{r}\left(1+(t \wedge 1)^{\left.-\frac{1}{m-1}\right), \quad t>0 .}\right.
$$

Proof. If we set $u:=z^{x}(\varphi)-\gamma_{\varphi}^{0}\left(z^{x}(\varphi)\right)$, we have

$$
u^{\prime}(t)=A u(t)+F\left(u(t)+\gamma_{\varphi}^{0}\left(z^{x}(\varphi)\right)(t)\right), \quad u(0)=x .
$$

If $\delta_{u}$ is the element of $\partial|u(t)|_{E}$ introduced in (2.13), with the notations used in the proof of Theorem 3.1 we have

$$
\begin{aligned}
\frac{d}{d t}^{-}|u(t)|_{E} & \leqslant\left\langle A u(t), \delta_{u}\right\rangle_{E}+\left\langle F\left(u(t)+\gamma_{\varphi}\left(z^{x}(\varphi)\right)(t)\right)-F\left(\gamma_{\varphi}\left(z^{x}(\varphi)\right)(t)\right), \delta_{u}\right\rangle_{E}+\left\langle F\left(\gamma_{\varphi}\left(z^{x}(\varphi)\right)(t)\right), \delta_{u}\right\rangle_{E} \\
& \leqslant-a|u(t)|^{m}+c\left(1+\left|\gamma_{\varphi}\left(z^{x}(\varphi)\right)(t)\right|_{E}^{m}\right) .
\end{aligned}
$$

Thus, thanks to Lemma 3.3, if $q$ is any constant as in (3.8) we obtain

$$
\frac{d^{-}}{d t}|u(t)|_{E} \leqslant-a|u(t)|^{m}+c(t)\left(1+\left|z^{x}\right|_{L^{q}(0, t ; E)}^{\gamma m}\right)|\varphi|_{L^{2}(0, t ; H)}^{m}+c
$$

for some continuous increasing function $c(t)$ vanishing at $t=0$. By a comparison argument proved in [1, Lemma 1.2.6] this gives

$$
|u(t)|_{E} \leqslant c t^{-\frac{1}{m-1}}+c(t)\left(1+\left|z^{x}(\varphi)\right|_{L^{q}(0, t ; E)}^{\gamma}\right)|\varphi|_{L^{2}(0, t ; H)}+c
$$

so that

$$
\begin{aligned}
\left|z^{x}(\varphi)(t)\right|_{E} & \leqslant|u(t)|_{E}+\left|\gamma_{\varphi}\left(z^{x}(\varphi)\right)(t)\right|_{E} \\
& \leqslant c t^{-\frac{1}{m-1}}+c(t)\left(1+\left|z^{x}(\varphi)\right|_{L^{q}(0, t ; E)}^{\gamma}\right)|\varphi|_{L^{2}(0, t ; H)}+c .
\end{aligned}
$$

Now, if (2.21) holds we can find $\bar{q} \geqslant 1$ fulfilling (3.8) such that $\bar{q} /(m-1)<1$. Hence, integrating with respect to $t \in[0, T]$ the $\bar{q}$ th power of both sides in (3.14), for any $\varphi \in L^{2}(0, \infty ; H)$, with $|\varphi|_{L^{2}(0, \infty ; H)} \leqslant r$, we get

$$
\begin{aligned}
\int_{0}^{T}\left|z^{x}(\varphi)(t)\right|_{E}^{\bar{q}} d t & \leqslant c \int_{0}^{T} t^{-\frac{\bar{q}}{m-1}} d t+c(T)\left(\int_{0}^{T}\left|z^{x}(\varphi)(t)\right|_{E}^{\bar{q}} d t\right)^{\gamma}|\varphi|_{L^{2}(0, T ; H)}^{\bar{q}}+c(T)\left(1+|\varphi|_{L^{2}(0, T ; H)}^{\bar{q}}\right) \\
& \leqslant c(T) r^{\bar{q}} \int_{0}^{T}\left|z^{x}(\varphi)(t)\right|_{E}^{\bar{q}} d t+c(T)\left(1+r^{\bar{q}}\right),
\end{aligned}
$$

for some continuous increasing function $c(t)$ vanishing at $t=0$. Thus, if we fix $T_{r}>0$ such that $c\left(T_{r}\right) r^{\bar{q}} \leqslant 1 / 2$, it follows

$$
\frac{1}{2} \int_{0}^{T_{r}}\left|z^{x}(\varphi)(t)\right|_{E}^{\bar{q}} d t \leqslant c\left(T_{r}\right)\left(1+r^{\bar{q}}\right),
$$

and going back to (3.14), for any $t \leqslant T_{r}$ this yields

$$
\sup _{x \in E}\left|z^{x}(\varphi)(t)\right|_{E} \leqslant c t^{-\frac{1}{m-1}}+c(t) r\left(1+c^{\gamma}\left(T_{r}\right)\left(1+r^{\bar{q}}\right)^{\gamma}\right)+c .
$$


Moreover, if $t>T_{r}$ we have $z^{x}(\varphi)(t)=z_{T_{r}}^{z^{x}(\varphi)\left(T_{r}\right)}(t)$ and then, due to (3.3)

$$
\left|z^{x}(\varphi)(t)\right|_{E} \leqslant c_{r}\left(1+\left|z^{x}(\varphi)\left(T_{r}\right)\right|_{E}\right), \quad t>T_{r} .
$$

Together with (3.15) this gives (3.13).

The next regularity result will be crucial in the proof of Proposition 5.4 which provides a characterization of the quasi-potential. We recall that in what follows we endow the space $C((-\infty ; 0] ; E)$ with the topology of uniform convergence on bounded sets $[-T, 0]$, for all $T>0$.

Lemma 3.5. Let $z_{0} \in C((-\infty, 0] ; E)$ solve the problem

$$
z_{0}(t)=\int_{-\infty}^{t} e^{(t-s) A} F\left(z_{0}(s)\right) d s+\int_{-\infty}^{t} e^{(t-s) A} G\left(z_{0}(s)\right) Q \varphi(s) d s, \quad t \leqslant 0,
$$

for some $\varphi \in L^{2}(-\infty, 0 ; H)$, and assume that

$$
\lim _{t \rightarrow-\infty}\left|z_{0}(t)\right|_{E}=0 \text {. }
$$

Then, under Hypotheses 1-5 and condition (2.14), if $\delta$ is the constant introduced in (2.15) we have that $z_{0} \in$ $L^{\infty}\left(-\infty, 0 ; D\left((-A)^{\delta+1 / 2}\right)\right)$ and

$$
\lim _{t \rightarrow-\infty}\left|z_{0}(t)\right|_{D\left((-A)^{\delta+1 / 2}\right)}=0 .
$$

Proof. For any $\varepsilon \in(0,1)$ we have

$$
\begin{aligned}
\left|\int_{-\infty}^{t} e^{(t-s) A} F\left(z_{0}(s)\right) d s\right|_{D\left((-A)^{\varepsilon}\right)} & \leqslant c \int_{-\infty}^{t} e^{-\alpha(t-s)}(t-s)^{-\varepsilon}\left|F\left(z_{0}(s)\right)\right|_{H} d s \\
& \leqslant c \int_{-\infty}^{t} e^{-\alpha(t-s)}(t-s)^{-\varepsilon} d s \sup _{s \leqslant t}\left|F\left(z_{0}(s)\right)\right|_{H} \\
& \leqslant c \sup _{s \leqslant t}\left|F\left(z_{0}(s)\right)\right|_{H},
\end{aligned}
$$

so that the mapping

$$
(-\infty, 0] \ni t \mapsto \int_{-\infty}^{t} e^{(t-s) A} F\left(z_{0}(s)\right) d s \in D\left((-A)^{\varepsilon}\right),
$$

belongs to $L^{\infty}\left(-\infty, 0 ; D\left((-A)^{\varepsilon}\right)\right)$. Moreover, since $F: E \rightarrow H$ is continuous, $F(0)=0$ and $\left|z_{0}(t)\right|_{E}$ goes to zero, as $t$ goes to $-\infty$, we have

$$
\lim _{t \rightarrow-\infty}\left|\int_{-\infty}^{t} e^{(t-s) A} F\left(z_{0}(s)\right) d s\right|_{D\left((-A)^{\varepsilon}\right)}=0, \quad \varepsilon \in(0,1) .
$$

Next, let $h \in L^{2}\left(-\infty, 0 ; D\left((-A)^{\gamma}\right)\right)$, for some $\gamma \geqslant 0$. We have

$$
(-A)^{\gamma+1 / 2} \int_{-\infty}^{t} e^{(t-s) A} h(s) d s=\sum_{k=1}^{\infty} \alpha_{k}^{\gamma+1 / 2} \int_{-\infty}^{t} e^{-(t-s) \alpha_{k}}\left\langle h(s), e_{k}\right\rangle_{H} d s e_{k},
$$


and then

$$
\begin{aligned}
\left|\int_{-\infty}^{t} e^{(t-s) A} h(s) d s\right|_{D\left((-A)^{\gamma+1 / 2)}\right.}^{2} & \left.=\sum_{k=1}^{\infty} \alpha_{k}^{2 \gamma+1}\left|\int_{-\infty}^{t} e^{-(t-s) \alpha_{k}}\right| h(s), e_{k}\right\rangle\left._{H} d s\right|^{2} \\
& \leqslant \sum_{k=1}^{\infty} \alpha_{k} \int_{-\infty}^{t} e^{-2(t-s) \alpha_{k}} d s \int_{-\infty}^{t} \alpha_{k}^{2 \gamma}\left|\left\langle h(s), e_{k}\right\rangle_{H}\right|^{2} d s \\
& \leqslant c \int_{-\infty}^{t} \sum_{k=1}^{\infty} \alpha_{k}^{2 \gamma}\left|\left\langle h(s), e_{k}\right\rangle_{H}\right|^{2} d s=c|h|_{L^{2}\left(-\infty, t ; D\left((-A)^{\gamma}\right)^{2}\right.} .
\end{aligned}
$$

Since $G\left(z_{0}\right) Q \varphi \in L^{2}(-\infty, 0 ; H)$ and for $t \leqslant 0$

$$
\left|G\left(z_{0}\right) Q \varphi\right|_{L^{2}(-\infty, t ; H)} \leqslant c\left(1+\sup _{s \leqslant t}\left|z_{0}(s)\right|_{E}\right)|Q \varphi|_{L^{2}(-\infty, t ; H)},
$$

by taking $\gamma=0$ and $h=G\left(z_{0}\right) Q \varphi$ in (3.19), we get

$$
\lim _{t \rightarrow-\infty}\left|\int_{-\infty}^{t} e^{(t-s) A} G\left(z_{0}(s)\right) Q \varphi(s) d s\right|_{D\left((-A)^{1 / 2}\right)}=0 .
$$

Thanks to (3.18) (with $\varepsilon=1 / 2)$ this implies that $z_{0} \in L^{\infty}\left(-\infty, 0 ; D\left((-A)^{1 / 2}\right)\right)$ and

$$
\lim _{t \rightarrow-\infty}\left|z_{0}(t)\right|_{D\left((-A)^{1 / 2}\right)}=0 \text {. }
$$

In particular, according to the characterization of $D\left((-A)^{1 / 2}\right)$ given in Proposition 2.1 this means that $z_{0} \in$ $L^{\infty}\left(-\infty, 0 ; H_{\mathcal{B}_{1 / 2}}^{1,2}\left(\mathcal{O} ; \mathbb{R}^{r}\right)\right)$. Then, since $f \in C^{\infty}\left(\overline{\mathcal{O}} \times \mathbb{R}^{r} ; \mathbb{R}^{r}\right)$, from [12, Theorem 5.5.4.1] we obtain that $F\left(z_{0}\right) \in L^{\infty}\left(-\infty, 0 ; H^{1,2}\left(\mathcal{O} ; \mathbb{R}^{r}\right)\right)$ and

$$
\sup _{t \leqslant 0}\left|F\left(z_{0}(t)\right)\right|_{H^{1,2}\left(\mathcal{O} ; \mathbb{R}^{r}\right)} \leqslant c \sup _{t \leqslant 0}\left|z_{0}(t)\right|_{H^{1,2}\left(\mathcal{O} ; \mathbb{R}^{r}\right)}\left(1+\sup _{t \leqslant 0}\left|z_{0}(t)\right|_{E}^{p}\right),
$$

for some $p \geqslant 1$. Moreover, as $z_{0}(t) \in H_{\mathcal{B}_{1 / 2}}^{1,2}\left(\mathcal{O} ; \mathbb{R}^{r}\right)$, for $t \leqslant 0$, we have that $\mathcal{B}_{1 / 2} z_{0}(t)=0$ on $\partial \mathcal{O}$ and then, thanks to assumption (2.17), we have that $\mathcal{B}_{1 / 2} F\left(z_{0}(t)\right)=0$ on $\partial \mathcal{O}$. This means that $F\left(z_{0}(t)\right) \in H_{\mathcal{B}_{1 / 2}}^{1,2}\left(\mathcal{O} ; \mathbb{R}^{r}\right)$, for $t \leqslant 0$, and hence, by using again Proposition 2.1 we have $F\left(z_{0}\right) \in L^{\infty}\left(-\infty, 0 ; D\left((-A)^{1 / 2}\right)\right)$. By proceeding as in the proof of (3.18), due to (3.21) this yields

$$
\lim _{t \rightarrow-\infty}\left|\int_{-\infty}^{t} e^{(t-s) A} F\left(z_{0}(s)\right) d s\right|_{D\left((-A)^{\varepsilon+1 / 2}\right)}=0, \quad \varepsilon<1 .
$$

By repeating these arguments we can conclude that for any $\gamma \geqslant 0$ and $\varepsilon<1$

$$
\lim _{t \rightarrow-\infty}\left|z_{0}(t)\right|_{D\left((-A)^{\gamma}\right)}=0 \Rightarrow \lim _{t \rightarrow-\infty}\left|\int_{-\infty}^{t} e^{(t-s) A} F\left(z_{0}(s)\right) d s\right|_{D\left((-A)^{\varepsilon+\gamma}\right)}=0 .
$$

Next we notice that with the same arguments used for $F\left(z_{0}\right)$ it is possible to prove that

$$
g\left(\cdot, z_{0}\right) \in L^{\infty}\left(-\infty, 0 ; H^{1,2}\left(\mathcal{O} ; \mathcal{L}\left(\mathbb{R}^{r}\right)\right)\right) .
$$


Moreover, as proved in [12, Theorem 4.6.1.1], if $s_{1} \leqslant s_{2}$ and $s_{1}+s_{2}>0$

$$
s_{2}>\frac{d}{2} \Rightarrow H^{s_{1}, 2}\left(\mathcal{O} ; \mathbb{R}^{r}\right) \cdot H^{s_{2}, 2}\left(\mathcal{O} ; \mathbb{R}^{r}\right) \hookrightarrow H^{s_{1}, 2}\left(\mathcal{O} ; \mathbb{R}^{r}\right)
$$

and

$$
s_{2}<\frac{d}{2} \Rightarrow H^{s_{1}, 2}\left(\mathcal{O} ; \mathbb{R}^{r}\right) \cdot H^{s_{2}, 2}\left(\mathcal{O} ; \mathbb{R}^{r}\right) \hookrightarrow H^{s_{1}+s_{2}-\frac{d}{2}, 2}\left(\mathcal{O} ; \mathbb{R}^{r}\right) .
$$

By using these embedding results we can study the regularity of the product $G\left(z_{0}\right) Q \varphi$ (and hence of the second integral in (3.16)). To this purpose we consider separately three different cases.

Case $d=1$ and $2 \delta \leqslant 1$. Since $Q \varphi(t) \in D\left((-A)^{\delta}\right)=H_{\mathcal{B}_{\delta}}^{2 \delta, 2}\left(\mathcal{O} ; \mathbb{R}^{r}\right)$, for $t \leqslant 0$, as $1>1 / 2=d / 2$, due to (3.23) and (3.24) we have

$$
G\left(z_{0}(t)\right) Q \varphi(t) \in H^{2 \delta, 2}\left(\mathcal{O} ; \mathbb{R}^{r}\right), \quad t \leqslant 0
$$

and

$$
\left|G\left(z_{0}(t)\right) Q \varphi(t)\right|_{H^{2 \delta, 2}\left(\mathcal{O} ; \mathbb{R}^{r}\right)} \leqslant c\left|g\left(\cdot, z_{0}(t, \cdot)\right)\right|_{H^{1,2}\left(\mathcal{O} ; \mathcal{L}\left(\mathbb{R}^{r}\right)\right)}|Q \varphi(t)|_{H^{2 \delta, 2}\left(\mathcal{O} ; \mathbb{R}^{r}\right)}
$$

Moreover, since $\mathcal{B}_{1 / 2} z_{0}(t)=0$ and $\mathcal{B}_{\delta}(Q \varphi(t))=0$ on $\partial \mathcal{O}$, for any $t \leqslant 0$, according to assumption (2.17) we have $\mathcal{B}_{\delta}\left(G\left(z_{0}(t)\right) Q \varphi(t)\right)=0$ on $\partial \mathcal{O}$, for any $t \leqslant 0$, so that $G\left(z_{0}\right) Q \varphi \in L^{2}\left(-\infty, 0 ; H_{\mathcal{B}_{\delta}}^{2 \delta, 2}\left(\mathcal{O} ; \mathbb{R}^{r}\right)\right)=$ $L^{2}\left(-\infty, 0 ; D\left((-A)^{\delta}\right)\right)$. Thanks to (3.19), with $h=G\left(z_{0}\right) Q \varphi$ and $\gamma=\delta$, this implies

$$
\lim _{t \rightarrow-\infty}\left|\int_{-\infty}^{t} e^{(t-s) A} G\left(z_{0}(s)\right) Q \varphi(s) d s\right|_{D\left((-A)^{\delta+1 / 2}\right)}=0
$$

so that, as (3.22) holds (with $\gamma=1 / 2$ and $\varepsilon=\delta$ ), we obtain (3.17).

Case $d \geqslant 1$ and $2 \delta>d / 2 \vee 1$. In this case, with the same arguments used above, we have that the mapping

$$
t \mapsto \int_{-\infty}^{t} e^{(t-s) A} G\left(z_{0}(s)\right) Q \varphi(s) d s
$$

belongs to $L^{2}(-\infty, 0 ; D(-A))$ and then, proceeding as for the previous case, due to (3.22) we have that $z_{0} \in$ $L^{\infty}(-\infty, 0 ; D(-A))$.

Now, if $2 \delta \leqslant 2$, by using again (3.24), we have $G\left(z_{0}\right) Q \varphi \in L^{2}\left(-\infty, 0 ; H_{\mathcal{B}_{\delta}, 2}^{2 \delta}\left(\mathcal{O} ; \mathbb{R}^{r}\right)\right)$ and then we can conclude as in the case of $d=1$ and $2 \delta \leqslant 1$. Otherwise, if $2 \delta>2$ we use again (3.24) and we obtain $G\left(z_{0}\right) Q \varphi \in$ $L^{2}\left(-\infty, 0 ; H_{\mathcal{B}_{1}}^{2,2}\left(\mathcal{O} ; \mathbb{R}^{r}\right)\right)$, so that $z_{0} \in L^{\infty}\left(-\infty, 0 ; D\left((-A)^{3 / 2}\right)\right)$. If $2 \delta \leqslant 3$ we conclude as above. If not, we go on with these arguments and in a finite number of steps we get $z_{0} \in L^{\infty}\left(-\infty, 0 ; D\left((-A)^{k / 2}\right)\right)$, for some $k \geqslant 2 \delta$ and hence we can conclude.

Case $d \geqslant 2$ and $2 \delta \leqslant d / 2$. Due to (2.16) we can fix $\varepsilon \in(0, \delta-(d-2) / 4)$. As $1 \leqslant d / 2$, by using (3.25) (with $1-\varepsilon$ and $2 \delta-\varepsilon$ ) we have that

$$
G\left(z_{0}\right) Q \varphi \in L^{2}\left(-\infty, 0 ; H_{\mathcal{B}_{\delta-\varepsilon-d / 4+1 / 2}}^{2(\delta-\varepsilon-d / 4)+1,2}\left(\mathcal{O} ; \mathbb{R}^{r}\right)\right),
$$

so that $z_{0} \in L^{\infty}\left(-\infty, 0 ; D\left((-A)^{\delta-\varepsilon-d / 4+1}\right)\right)$.

If $\delta-\varepsilon-d / 4+1>d / 4$, by using (3.24) we obtain $G\left(z_{0}\right) Q \varphi \in L^{2}\left(-\infty, 0 ; H_{B_{\delta}}^{2 \delta, 2}\left(\mathcal{O} ; \mathbb{R}^{r}\right)\right)$ and then we can conclude as above (see the case of $d=1$ and $2 \delta \leqslant 1$ ).

If $\delta-\varepsilon-d / 4+1 \leqslant d / 4$, then, by using again (3.25) (with $2 \delta-3 \varepsilon-d / 2+2$ and $2 \delta-\varepsilon$ ) we easily obtain

$$
\left.G\left(z_{0}\right) Q \varphi \in L^{2}\left(-\infty, 0 ; H_{\mathcal{B}_{2(\delta-\varepsilon-d / 4)+1}(\delta-\varepsilon)}^{(\mathcal{O} ; / 4)+2} \mathbb{R}^{r}\right)\right),
$$


so that $z_{0} \in L^{\infty}\left(-\infty, 0 ; D\left((-A)^{2(\delta-\varepsilon-d / 4)+3 / 2}\right)\right)$. If $2(\delta-\varepsilon-d / 4)+3 / 2>d / 4$, we can conclude as above. Otherwise we repeat the same arguments a finite number of times and we get

$$
z_{0} \in L^{\infty}\left(-\infty, 0 ; D\left((-A)^{k(\delta-\varepsilon-d / 4)+(k+1) / 2}\right)\right),
$$

for some $k \in \mathbb{N}$ such that $k(\delta-\varepsilon-d / 4)+(k+1) / 2>d / 4$ (and this is possible as $\varepsilon<\delta-(d-2) / 4)$. At this point we conclude as above.

Finally, we consider the uncontrolled version of Eq. (3.2), namely

$$
z^{\prime}(t)=A z(t)+F(z(t)), \quad z(T)=x .
$$

With the notations introduced at the beginning of this section its solution will be denoted by $z_{T}^{x}(0)$.

Proposition 3.6. Under Hypotheses 1-4, for any $R>0$

$$
\lim _{t-T \rightarrow \infty} \sup _{|x|_{E} \leqslant R}\left|z_{T}^{x}(0)(t)\right|_{E}=0 .
$$

Proof. If $\delta_{z(t)}$ is the element of $\partial\left|z_{T}^{x}(0)(t)\right|_{E}$ introduced in (2.12), we have

$$
\frac{d}{d t}^{-}\left|z_{T}^{x}(0)(t)\right|_{E} \leqslant\left\langle A z_{T}^{x}(0)(t), \delta_{z(t)}\right\rangle_{E}+\left\langle F\left(z_{T}^{x}(0)(t)\right), \delta_{z(t)}\right\rangle_{E} \leqslant-\alpha\left|z_{T}^{x}(0)(t)\right|_{E} .
$$

By comparison this yields

$$
\left|z_{T}^{x}(0)(t)\right|_{E} \leqslant e^{-\alpha(t-T)}|x|_{E}, \quad t \geqslant T,
$$

so that (3.27) follows.

\section{A non-linear local exact controllability problem}

Since we are dealing with space dimension $d \geqslant 1$, we cannot assume in general the operator $Q$ to be invertible and then the proof of compactness of the level sets of the quasi-potential associated with system (1.1) is more delicate than in the classical non-degenerate case (see [14]). For later use, in this section we prove some preliminary results about the local exact controllability of the skeleton system (3.2). Such results will be crucial in the proof of the characterization of the quasi-potential given in Proposition 5.4.

We start with a few definitions about exact and local exact controllability.

\section{Definition 4.1.}

Let

$$
z^{\prime}(t)=H(z)(t)+K(z, \varphi)(t), \quad z(0)=0,
$$

be some controlled system, with state space $V$ and control space $U$, and let $z(\varphi)$ denote the solution corresponding to the control $\varphi$.

(1) The system is exactly controllable at time $T>0$ if for any state $x \in V$ there exists an admissible control $\varphi \in U$ such that $z(\varphi)(T)=x$.

(2) The system is locally exactly controllable at time $T>0$ if there exists $\varepsilon>0$ such that for any $x \in V$, with $|x|_{V}<\varepsilon$, there exists an admissible control $\varphi \in U$ such that $z(\varphi)(T)=x$. 
Here, in addition to the non-linear control problem

$$
z^{\prime}(t)=A z(t)+F(z(t))+G(z(t)) Q \varphi(t), \quad z(0)=0,
$$

for any $\psi \in L^{2}(0, T ; H)$ we consider the linearized problem

$$
y^{\prime}(t)=\left[A+F^{\prime}(0)\right] y(t)+G(0) Q \psi(t), \quad y(0)=0 .
$$

In what follows we shall denote the solution of (4.2) by $y^{\psi}$. As $y^{\psi} \in L^{2}\left(0, T ; D\left((-A)^{\delta}\right)\right)$ and $f^{\prime}(\xi, 0) \in$ $C^{\infty}\left(\mathcal{O} ; \mathcal{L}\left(\mathbb{R}^{r}\right)\right)$, with the arguments used in the proof of Lemma 3.5 , it is immediate to show that $y^{\psi} \in$ $L^{\infty}\left(0, T ; D\left((-A)^{\delta+1 / 2}\right)\right)$. Moreover, if we fix $T>0$ and denote by $L_{T}$ the mapping

$$
L_{T}: L^{2}(0, T ; H) \rightarrow D\left((-A)^{\delta+1 / 2}\right), \quad \psi \mapsto L_{T} \psi:=y^{\psi}(T),
$$

it is clearly continuous. Now, if we show that there exists $c_{T}>0$ such that for any $h \in D\left((-A)^{\delta+1 / 2}\right)$

$$
\left|L_{T}^{\star} h\right|_{L^{2}(0, T ; H)} \geqslant c_{T}|h|_{D\left((-A)^{\delta+1 / 2}\right)},
$$

we have that for any $h \in D\left((-A)^{\delta+1 / 2}\right)$ there exists $\psi \in L^{2}(0, T ; H)$ such that $y^{\psi}(T)=h$, so that the linear system (4.2) with state space $V:=D\left((-A)^{\delta+1 / 2}\right)$ and control space $U:=L^{2}(0, T ; H)$ is exactly controllable at time $T>0$.

It is immediate to check that

$$
L_{T}^{\star} h(s)=Q[G(0)]^{\star} e^{(T-s)\left[A+F^{\prime}(0)\right]^{\star}} h, \quad s \in[0, T] .
$$

Then, due to (2.14) we have

$$
\begin{aligned}
\left|L_{T^{k}}^{\star} h\right|_{L^{2}(0, T ; H)}^{2} & =\int_{0}^{T}\left|Q[G(0)]^{\star} e^{(T-s)\left[A+F^{\prime}(0)\right]^{\star}} h\right|_{H}^{2} d s \geqslant c(T) \int_{0}^{T}\left|Q e^{(T-s) A} h\right|_{H}^{2} d s \\
& =c(T) \int_{0}^{T} \sum_{k=1}^{\infty} \lambda_{k}^{2} e^{-2 \alpha_{k}(T-s)} h_{k}^{2} d s,
\end{aligned}
$$

with $h_{k}=\left\langle h, e_{k}\right\rangle_{H}$. Thanks to (2.15) this gives

$$
\begin{aligned}
\left|L_{T}^{\star} h\right|_{L^{2}(0, T ; H)}^{2} & \geqslant c(T) \sum_{k=1}^{\infty} \alpha_{k}^{-2 \delta} h_{k}^{2} \int_{0}^{T} e^{-2 \alpha_{k}(T-s)} d s=c(T) \sum_{k=1}^{\infty} \alpha_{k}^{-2 \delta} h_{k}^{2} \frac{\left(1-e^{-2 \alpha_{k} T}\right)}{2 \alpha_{k}} \\
& \geqslant c(T)\left(1-e^{-2 \alpha T}\right) \sum_{k=1}^{\infty} \alpha_{k}^{-2(\delta+1 / 2)} h_{k}^{2}=c(T)\left(1-e^{-2 \alpha T}\right)|h|_{D\left((-A)^{\delta+1 / 2}\right)}^{2},
\end{aligned}
$$

so that (4.3) follows with $C_{T}:=c(T)\left(1-e^{-2 \alpha T}\right)$.

Now, since the mapping $L_{T}: L^{2}(0, T ; H) \rightarrow D\left((-A)^{\delta+1 / 2}\right)$ is surjective and continuous, by general arguments we can define its pseudo-inverse $S_{T}$ at a point $x \in D\left((-A)^{\delta+1 / 2}\right)$ as the unique $\psi \in L^{2}(0, T ; H)$ such that

$$
L_{T} \psi=x, \quad\langle\psi-\varphi, \psi\rangle_{L^{2}(0, T ; H)}=0, \quad \text { for all } \varphi \in L^{2}(0, T ; H) \text { with } L_{T} \varphi=x .
$$

Equivalently $\psi=S_{T} x$ is the element of smallest norm satisfying $L_{T} \psi=x$. We note that the operator $S_{T}: D\left((-A)^{\delta+1 / 2}\right) \rightarrow L^{2}(0, T ; H)$ is linear and

$$
\left\|S_{T}\right\|_{\mathcal{L}\left(D\left((-A)^{\delta+1 / 2}\right) ; L^{2}(0, T ; H)\right)} \leqslant c_{T}^{-1} .
$$

This allows us to prove the local exact controllability of the non-linear system (4.1). 
Theorem 4.2. There exists $T_{0}>0$ such that system (4.1) is locally exactly controllable, with state space $V:=$ $D\left((-A)^{\delta+1 / 2}\right)$ and control space $U:=L^{2}(0, T ; H)$, for any $T \leqslant T_{0}$.

\section{Proof.}

For any $x \in V$ we consider the problem

$$
z^{\prime}(t)=A z(t)+F(z(t))+G(z(t)) Q S_{T} x(t), \quad z(0)=0,
$$

whose solution $z^{0}\left(S_{T} x\right)$ at time $t$ is denoted by $\Gamma_{t}(x)$. Proceeding as in the proof of Lemma 3.5, it is possible to prove that $z^{0}\left(S_{T} x\right) \in L^{\infty}(0, T ; V)$, so that $\Gamma_{t}$ maps $V$ into $V$.

If we show that there exists some $T_{0}>0$ such that $\Gamma_{T}$ is differentiable in a neighborhood of zero, for any $T \leqslant T_{0}$, and $D \Gamma_{T}(0)=I$, by the local inversion theorem we have that there exist two neighborhoods $U_{1}$ and $U_{2}$ of 0 in $V$ such that $\Gamma_{T}: U_{1} \rightarrow U_{2}$ is invertible. Due to the definition of $\Gamma_{T}$, this means that for any $y \in U_{2}$ there exists $x=\Gamma_{T}^{-1}(y) \in U_{1}$ such that

$$
y=\int_{0}^{T} e^{(T-s) A} F\left(z^{0}\left(S_{T} x\right)(s)\right) d s+\int_{0}^{T} e^{(T-s) A} G\left(z^{0}\left(S_{T} x\right)(s)\right) Q S_{T} x(s) d s,
$$

so that $\varphi^{y}:=S_{T} \Gamma_{T}^{-1}(y)$ is the control such that $z^{0}\left(\varphi^{y}\right)(T)=y$.

For any $x \in V$ we have that $z^{0}\left(S_{T} x\right)$ is the unique fixed point of the mapping $\mathcal{F}_{T}: V \times L^{\infty}(0, T ; V) \rightarrow$ $L^{\infty}(0, T ; V)$ defined by

$$
\mathcal{F}_{T}(x, z)(t):=\int_{0}^{t} e^{(t-s) A} F(z(s)) d s+\int_{0}^{t} e^{(t-s) A} G(z(s)) Q S_{T} x(s) d s .
$$

We denote such fixed point by $z(x)$. Notice that, proceeding as in the proof of Lemma 3.5, due to (3.19) and (4.4), we have

$$
\left|\mathcal{F}_{T}(x, z)\right|_{L^{\infty}(0, T ; V)} \leqslant c T|z|_{L^{\infty}(0, T ; V)}\left(1+|z|_{L^{\infty}(0, T ; V)}^{p}\right)+c c_{T}^{-1}\left(1+|z|_{L^{\infty}(0, T ; V)}^{p}\right)|x|_{V},
$$

for some $p \geqslant 1$. Thus, if we fix $T \leqslant T_{1}:=1 / 4 c$ and $R_{T} \leqslant c_{T} / 4 c$, we get

$$
|x|_{V} \leqslant R_{T}, \quad|z|_{L^{\infty}(0, T ; V)} \leqslant 1 \Rightarrow\left|\mathcal{F}_{T}(x, z)\right|_{L^{\infty}(0, T ; V)} \leqslant 1
$$

so that $\mathcal{F}_{T}(x, \cdot)$ maps $B_{L^{\infty}(0, T ; V)}(1)$ into itself, for any $x \in B_{V}\left(R_{T}\right)$.

It is immediate to check that for any fixed $z \in L^{\infty}(0, T ; V)$ the mapping

$$
\mathcal{F}_{T}(\cdot, z): V \rightarrow L^{\infty}(0, T ; V), \quad x \mapsto \mathcal{F}_{T}(x, z),
$$

is Fréchet differentiable and for any $x, h \in V$

$$
\left[\frac{\partial \mathcal{F}_{T}}{\partial x}(x, z) h\right](t)=\int_{0}^{t} e^{(t-s) A} G(z(s)) Q S_{T} h(s) d s, \quad t \in[0, T] .
$$

Since $2 \delta+1>d / 2$, we have $H^{2 \delta+1,2}\left(\mathcal{O} ; \mathbb{R}^{r}\right) \subset L^{\infty}\left(\mathcal{O} ; \mathbb{R}^{r}\right)$ and then, as shown in [12, Theorem 5.5.3.1], the Nemytskij composition operator $F$ is differentiable in $H^{2 \delta+1,2}\left(\mathcal{O} ; \mathbb{R}^{r}\right)$ and for any $x, y \in H^{2 \delta+1,2}\left(\mathcal{O} ; \mathbb{R}^{r}\right)$

$$
\left[F^{\prime}(x) y\right](\xi)=D_{\sigma} f(\xi, x(\xi)) y(\xi), \quad \xi \in \overline{\mathcal{O}} .
$$

Notice that due to (2.18) if $x, y \in D\left((-A)^{\delta}\right)$ then $\mathcal{B}_{\delta}\left[F^{\prime}(x) y\right]=0$ on $\partial O$, so that $F^{\prime}(x) y \in D\left((-A)^{\delta}\right)$. In particular, since $2 \delta+1>d / 2$, by using (3.24) for any $x, y \in V$ we have

$$
\left|F^{\prime}(x) y\right|_{D\left((-A)^{\delta}\right)} \leqslant c\left(1+|x|_{V}^{p}\right)|y|_{V}
$$


for some constant $p \geqslant 1$. Moreover, due to (3.24) and to the boundary assumptions (2.17) for any $x \in V$ and $h \in D\left((-A)^{\delta}\right)$ we have that $G(x) h \in D\left((-A)^{\delta}\right)$. Then by using again the result proved in [12, Theorem 5.5.3.1] we have that the mapping $x \in H^{2 \delta+1,2}\left(\mathcal{O} ; \mathbb{R}^{r}\right) \mapsto G(x) h \in H^{2 \delta, 2}\left(\mathcal{O} ; \mathbb{R}^{r}\right)$ is differentiable and for any $y \in H^{2 \delta+1,2}\left(\mathcal{O} ; \mathbb{R}^{r}\right)$

$$
\left(\left[G^{\prime}(x) h\right] y\right)(\xi)=[D(G(\cdot) h)(x) y](\xi)=\left[D_{\sigma} g(\xi, x(\xi)) y(\xi)\right] h(\xi), \quad \xi \in \overline{\mathcal{O}} .
$$

In particular, if $x, y \in V$ and $h \in D\left((-A)^{\delta}\right)$ due to (2.18) we have that $\left[G^{\prime}(x) h\right] y \in D\left((-A)^{\delta}\right)$ and

$$
\left|\left[G^{\prime}(x) h\right] y\right|_{D\left((-A)^{\delta}\right)} \leqslant c\left(1+|x|_{V}^{p}\right)|y|_{V}|h|_{D\left((-A)^{\delta}\right)},
$$

for some constant $p \geqslant 1$.

Thus, thanks to (3.19) and to (4.6) and (4.8) it is not difficult to show that for any fixed $x \in V$ the mapping

$$
z \in L^{\infty}(0, T ; V) \mapsto \mathcal{F}_{T}(x, z) \in L^{\infty}(0, T ; V)
$$

is differentiable and for any $x \in V$ and $z, w \in L^{\infty}(0, T ; V)$ it holds

$$
\left[\frac{\partial \mathcal{F}_{T}}{\partial z}(x, z) w\right](t)=\int_{0}^{t} e^{(t-s) A} F^{\prime}(z(s)) w(s) d s+\int_{0}^{t} e^{(t-s) A}\left[G^{\prime}(z(s)) w(s)\right] Q S_{T} x(s) d s .
$$

Moreover, according to (3.19) and (4.4) and to (4.7) and (4.9) we have

$$
\begin{aligned}
\left|\left[\frac{\partial \mathcal{F}_{T}}{\partial z}(x, z) w\right](t)\right|_{V} & \leqslant c\left(\left|F^{\prime}(z) w\right|_{L^{2}\left(0, t ; D\left((-A)^{\delta}\right)\right)}+\left|\left[G^{\prime}(z) w\right] Q S_{T} x\right|_{L^{2}\left(0, t ; D\left((-A)^{\delta}\right)\right)}\right) \\
& \leqslant c\left[\int_{0}^{t}\left(1+|z|_{V}^{p}\right)^{2}\left(1+\left|S_{T} x(s)\right|_{D\left((-A)^{\delta}\right)}^{2}\right)|w(s)|_{V}^{2} d s\right]^{1 / 2} \\
& \leqslant c^{\prime}\left(1+|z|_{L^{\infty}(0, t ; V)}^{p}\right)\left(\sqrt{t}+c_{T}^{-1}|x|_{V}\right)|w|_{L^{\infty}(0, t ; V)} .
\end{aligned}
$$

Hence, if $T \leqslant T_{1},|z|_{L^{\infty}(0, T ; V)} \leqslant 1$ and $|x|_{V} \leqslant R$, for some $R \leqslant R_{T}$, we get

$$
\left|\left[\frac{\partial \mathcal{F}_{T}}{\partial z}(x, z) w\right]\right|_{V} \leqslant 2 c^{\prime}\left(\sqrt{T}+c_{T}^{-1} R\right)|w|_{L^{\infty}(0, T ; V)} .
$$

This means that if we fix $0<\alpha<1$ and $T_{0}:=\left(\alpha / 4 c^{\prime}\right)^{2} \wedge T_{1}$, for any $T \leqslant T_{0}$ and any $R_{T} \leqslant c_{T}\left(\alpha / c^{\prime} \wedge 1 / c\right) / 4$ we have

$$
|x|_{V} \leqslant R_{T}, \quad|z|_{L^{\infty}(0, T ; V)} \leqslant 1 \Rightarrow\left|\frac{\partial \mathcal{F}_{T}}{\partial z}(x, z) w\right|_{{\mathcal{L}\left(L^{\infty}(0, T ; V), L^{\infty}(0, T ; V)\right)} \leqslant \alpha .}
$$

Thus from the theorem of contractions depending on parameters (see for example [1, Proposition C.0.3] for a proof in this setting), we have that for any $T \leqslant T_{0}$ the mapping

$$
x \in B_{V}\left(R_{T}\right) \mapsto z(x) \in L^{\infty}(0, T ; V),
$$

is differentiable. Moreover, for any $x \in B_{V}\left(R_{T}\right), h \in V$ and $t \in[0, T]$

$$
\begin{aligned}
{[D z(x) h](t)=D \Gamma_{t}(x) h=} & \int_{0}^{t} e^{(t-s) A} F^{\prime}\left(\Gamma_{S}(x)\right) D \Gamma_{S}(x) h d s+\int_{0}^{t} e^{(t-s) A}\left[G^{\prime}\left(\Gamma_{S}(x)\right) D \Gamma_{s}(x) h\right] Q S_{T} x(s) d s \\
& +\int_{0}^{t} e^{(t-s) A} G\left(\Gamma_{S}(x)\right) Q S_{T} h(s) d s .
\end{aligned}
$$


Since $F(0)=0$ and $S_{T} 0=0$, we clearly have $z(0)=0$ and $\Gamma_{s}(0)=0$, for any $s \in[0, T]$. This implies that

$$
D \Gamma_{t}(0) h=\int_{0}^{t} e^{(t-s) A} F^{\prime}(0) D \Gamma_{s}(0) h d s+\int_{0}^{t} e^{(t-s) A} G(0) Q S_{T} h(s) d s .
$$

Therefore, by a uniqueness argument we have that $v(t):=D \Gamma_{t}(0) h$ is the solution of

$$
v^{\prime}(t)=\left[A+F^{\prime}(0)\right] v(t)+G(0) Q S_{T} h(t), \quad v(0)=0 .
$$

This means that $D \Gamma_{T}(0) h=v(T)=h$, so that $D \Gamma_{T}(0)=I$.

\section{Compactness of level sets of the quasi-potential}

For any $t_{1}<t_{2}$ and $z \in C\left(\left[t_{1}, t_{2}\right] ; E\right)$ we define

$$
I_{t_{1}, t_{2}}(z):=\frac{1}{2} \inf \left\{|\varphi|_{L^{2}\left(t_{1}, t_{2} ; H\right)}^{2} ; z=z(\varphi)\right\},
$$

where $z(\varphi)$ is the solution of the skeleton equation (3.2) in the interval $\left[t_{1}, t_{2}\right]$, corresponding to the control $\varphi$ (with the usual convention that inf $\emptyset=+\infty$ ). For simplicity of notations, when $t_{1}=0$ and $t_{2}=t>0$ we shall write $I_{t}$ and when $t_{2}=0$ and $t_{1}=-t<0$ we shall write $I_{-t}$.

In [3, Theorem 5.1] we have proved that for any $x \in E, r \geqslant 0$ and $t_{1}<t_{2}$ the level set

$$
K_{x, t_{1}, t_{2}}(r):=\left\{z \in C\left(\left[t_{1}, t_{2}\right] ; E\right) ; z\left(t_{1}\right)=x, I_{t_{1}, t_{2}}(z) \leqslant r\right\}
$$

is compact. In fact, it is not difficult to adapt the proof of [3, Theorem 5.1] in order to show that for any compact set $\Lambda \subset E$ the level set

$$
K_{\Lambda, t_{1}, t_{2}}(r):=\left\{z \in C\left(\left[t_{1}, t_{2}\right] ; E\right) ; z\left(t_{1}\right) \in \Lambda, I_{t_{1}, t_{2}}(z) \leqslant r\right\}
$$

is compact. Notice that in what follows, if $t_{1}=0$ and $t_{2}=t$ we shall write $K_{x, t}(r)$ and $K_{\Lambda, t}(r)$ instead of $K_{x, 0, t}(r)$ and $K_{\Lambda, 0, t}(r)$.

Analogously, for any $z \in C((-\infty ; 0] ; E)$ we define

$$
I_{-\infty}(z):=\frac{1}{2} \inf \left\{|\varphi|_{L^{2}(-\infty, 0 ; H)}^{2} ; z=z(\varphi)\right\}
$$

and for any $r \geqslant 0$

$$
K_{-\infty}(r):=\left\{z \in C((-\infty ; 0] ; E) ; I_{-\infty}(z) \leqslant r, \lim _{t \rightarrow-\infty}|z(t)|_{E}=0\right\} .
$$

We note that for any $z \in C((-\infty ; 0] ; E)$

$$
I_{-\infty}(z)=\sup _{t \geqslant 0} I_{-t}(z)
$$

Finally, for any $x \in E$ we define the quasi-potential

$$
V(x):=\inf \left\{I_{t}(z) ; t>0, z \in C([0, t] ; E) \text {, with } z(0)=0 \text { and } z(t)=x\right\} .
$$

In this section we shall prove that the level sets of $V$ are compact, so that $V$ is an admissible action functional for the large deviations estimates of the family of invariant measures $\left\{v_{\varepsilon}\right\}_{\varepsilon>0}$.

First of all we notice that $x=0$ is the unique minimum point of $V$, i.e.

$$
V(x)=0 \Leftrightarrow x=0 .
$$


Actually, if $x=0$ then clearly $V(x)=0$. On the other side, if $V(x)=0$ for any $\varepsilon>0$ there exist $T_{\varepsilon}>0$ and $z_{\varepsilon} \in C\left(\left[0, T_{\varepsilon}\right] ; E\right)$ such that $z_{\varepsilon}(0)=0$ and $z_{\varepsilon}\left(T_{\varepsilon}\right)=x$ and $I_{T_{\varepsilon}}\left(z_{\varepsilon}\right)<\varepsilon$. This means that for each $\varepsilon>0$ there exists $\varphi_{\varepsilon} \in L^{2}\left(0, T_{\varepsilon} ; H\right)$ such that

$$
z_{\varepsilon}=z_{0}^{0}\left(\varphi_{\varepsilon}\right) \quad \text { and } \quad \frac{1}{2}\left|\varphi_{\varepsilon}\right|_{L^{2}\left(0, T_{\varepsilon} ; H\right)}^{2} \leqslant 2 \varepsilon
$$

According to Proposition 3.2 this implies that

$$
\lim _{\varepsilon \rightarrow 0}\left|z_{\varepsilon}\left(T_{\varepsilon}\right)\right|_{E}=\lim _{\varepsilon \rightarrow 0}\left|z_{0}^{0}\left(\varphi_{\varepsilon}\right)\left(T_{\varepsilon}\right)\right|_{E}=0,
$$

and hence, as $z_{\varepsilon}\left(T_{\varepsilon}\right)=x$, we have that $x=0$.

Now, as we are assuming $F(0)=0$, if $G(0)=0$ then for each $t>0$ and $z \in C([0, t] ; E)$, with $z(0)=0$ and $z(t)=x \neq 0$, we clearly have $I_{t}(z)=+\infty$. Due to the arbitrariness of $t>0$, this means that

$$
G(0)=0 \Rightarrow V(x)= \begin{cases}+\infty & \text { if } x \neq 0 \\ 0 & \text { if } x=0 .\end{cases}
$$

In particular, if $G(0)=0$ the level sets of $V$ are trivially compact.

Our aim is to prove that the level sets of $V$ are compact in $E$, even under condition (2.14). We start by proving the compactness of the sets $K_{-\infty}(r)$.

Proposition 5.1. Assume Hypotheses $1-4$. Then for any $r \geqslant 0$ the set $K_{-\infty}(r)$ is compact in $C((-\infty, 0] ; E)$.

Proof. Given any sequence $\left\{z_{n}\right\} \subset K_{-\infty}(r)$, we have to show that there exists a subsequence $\left\{z_{n_{k}}\right\}$ converging in $C((-\infty, 0] ; E)$ to some $\hat{z} \in K_{-\infty}(r)$. For this purpose we need a preliminary result, whose proof is postponed.

Lemma 5.2. There exists $\theta_{\star} \in(0,1)$ such that for any $r \geqslant 0$

$$
I_{-\infty}(z) \leqslant r \quad \text { and } \quad \sup _{t \leqslant 0}|z(t)|_{E}<\infty \Rightarrow \sup _{t \leqslant 0}|z(t)|_{C^{\theta_{\star}\left(\overline{\mathcal{O}} ; \mathbb{R}^{r}\right)}} \leqslant L(r)
$$

for some constant $L(r)>0$.

Due to the previous lemma, if $z \in K_{-\infty}(r)$, for any $k \in \mathbb{N}$ the restriction of $z$ to the interval $[-k, 0]$ belongs to $K_{\Lambda,-k, 0}(r)$, where

$$
\Lambda:=\left\{x \in E ;|x|_{C^{\theta_{*}}\left(\overline{\mathcal{O}} ; \mathbb{R}^{r}\right)} \leqslant L(r)\right\} .
$$

As $\Lambda$ is compact in $E$, we have that $K_{\Lambda,-k, 0}(r)$ is compact in $C([-k, 0] ; E)$ for any $k \in \mathbb{N}$. Then by taking $k=1$ we can find $\left\{z_{n_{1}}\right\} \subseteq\left\{z_{n}\right\}$ and $\hat{z}_{1} \in C([-1,0] ; E)$ such that

$$
\lim _{n_{1} \rightarrow \infty} z_{n_{1}[-1,0]}=\hat{z}_{1}, \quad \text { in } C([-1,0] ; E) .
$$

In particular, as $I_{-1}$ is lower semi-continuous (see [3, Theorem 5.1]) we have $I_{-1}\left(\hat{z}_{1}\right) \leqslant r$. With the same arguments, we can find a subsequence $\left\{z_{n_{2}}\right\} \subseteq\left\{z_{n_{1}}\right\}$ and $\hat{z}_{2} \in C([-2,0] ; E)$ such that

$$
\lim _{n_{2} \rightarrow \infty} z_{\left.n_{2 \mid} \mid-2,0\right]}=\hat{z}_{2}, \quad \text { in } C([-2,0] ; E),
$$

and $I_{-2}\left(\hat{z}_{2}\right) \leqslant r$. Proceeding in this way, we can find a subsequence $\left\{z_{n^{\prime}}\right\} \subseteq\left\{z_{n}\right\}$ converging to some $\hat{z}$ in $C((-\infty, 0] ; E)$. By construction for each $k \in \mathbb{N}$ we have that $I_{-k}\left(z_{n^{\prime}}\right) \leqslant r$, for any $n^{\prime} \in \mathbb{N}$ large enough and then, due to the lower semi-continuity of $I_{-k}$, we have $I_{-k}(\hat{z}) \leqslant r$. This implies that $I_{-\infty}(\hat{z}) \leqslant r$. Moreover, it is immediate to check that

$$
\sup _{t \leqslant 0}|\hat{z}(t)|_{C^{\theta \star}\left(\overline{\mathcal{O}} ; \mathbb{R}^{r}\right)} \leqslant L(r) .
$$


Thus, in order to show that $\hat{z} \in K_{-\infty}(r)$, it remains to prove that

$$
\lim _{t \rightarrow-\infty}|\hat{z}(t)|_{E}=0 \text {. }
$$

If this is not true, there exist a constant $\eta>0$ and a sequence $\left\{t_{n}\right\}$ decreasing to $-\infty$ such that $\left|\hat{z}\left(t_{n}\right)\right|_{E} \geqslant \eta$, for any $n \in \mathbb{N}$. The next lemma, whose proof is postponed, shows that in fact this is not possible.

Lemma 5.3. There exist $t_{0}>0$ and $\beta>0$ such that

$$
|\hat{z}(t)|_{E} \geqslant \eta \Rightarrow I_{t-t_{0}, t}(\hat{z}) \geqslant \beta .
$$

Now, we can conclude the proof of the proposition. Actually, if we assume that $\left|\hat{z}\left(t_{n}\right)\right|_{E} \geqslant \eta$, due to the previous lemma there exists $t_{0}>0$ such that $I_{t_{n}-t_{0}, t_{n}}(\hat{z}) \geqslant \beta>0$, for any $n \in \mathbb{N}$. Thus, if we fix any subsequence $\left\{t_{n_{k}}\right\} \subseteq\left\{t_{n}\right\}$ such that $t_{n_{k+1}} \leqslant t_{n_{k}}-t_{0}$, for any $m \in \mathbb{N}$ we have

$$
I_{-\infty}(\hat{z}) \geqslant \sum_{k=1}^{m} I_{t_{n_{k+1}}, t_{n_{k}}}(\hat{z}) \geqslant \sum_{k=1}^{m} I_{t_{n_{k}}-t_{0}, t_{n_{k}}}(\hat{z}) \geqslant \beta m .
$$

Thus, as $m$ can be taken arbitrarily large, we get $I_{-\infty}(\hat{z})=+\infty$, which is not true.

Now, in order to conclude the proof of Proposition 5.1 it remains to prove Lemmas 5.2 and 5.3.

Proof of Lemma 5.2. Since $I_{-\infty}(z) \leqslant r$, for any $-T \leqslant t \leqslant 0$ we have

$$
z(t)=e^{(t+T) A} z(-T)+\int_{-T}^{t} e^{(t-s) A} F(z(s)) d s+\gamma_{\varphi}^{-T}(z)(t),
$$

for some $\varphi \in L^{2}(-T, 0 ; H)$, with $|\varphi|_{L^{2}(-T, 0 ; H)}^{2} \leqslant 3 r$. Then, using (3.5) and (3.6) we can find a constant $c_{r}$ not depending on $T$ such that for any $\xi, \eta \in \overline{\mathcal{O}}$ and $t \geqslant-T$

$$
\begin{aligned}
|z(t, \xi)-z(t, \eta)| \leqslant & \left|e^{(t+T) A} z(-T)(\xi)-e^{(t+T) A} z(-T)(\eta)\right| \\
& +c_{r}\left(1+\sup _{t \geqslant-T}|z(t)|_{E}^{m}+\sup _{t \geqslant-T}|z(t)|_{E}\right)|\xi-\eta|^{\theta_{\star}} .
\end{aligned}
$$

According to (2.9), with $\theta=0$, and to (3.3) this implies

$$
|z(t, \xi)-z(t, \eta)| \leqslant c e^{-\alpha(t+T)}|z(-T)|_{E}+c_{r}\left(1+|z(-T)|_{E}^{m}\right)|\xi-\eta|^{\theta_{\star}}
$$

and since $\sup _{t \leqslant 0}|z(t)|_{E}=: \kappa<\infty$, it follows that for any $t \geqslant-T$

$$
|z(t, \xi)-z(t, \eta)| \leqslant c e^{-\alpha(t+T)}|z(-T)|_{E}+c_{r}\left(1+\kappa^{m}\right)|\xi-\eta|^{\theta_{\star}} .
$$

By taking the limit above for $T$ tending to infinity, we obtain

$$
|z(t, \xi)-z(t, \eta)| \leqslant c_{r}\left(1+\kappa^{m}\right)|\xi-\eta|^{\theta_{\star}}, \quad t \leqslant 0,
$$

which implies the lemma.

Proof of Lemma 5.3. For any $s>0$, let $z_{t-s}^{x}(0)$ be the solution of problem (3.26) starting from $x$ at time $t-s$. If $\Lambda$ is the set introduced in (5.4), due to (3.27) there exists $t_{0}>0$ large enough such that

$$
\sup _{x \in \Lambda}\left|z_{t-t_{0}}^{x}(0)(t)\right|_{E} \leqslant \frac{\eta}{2} \text {. }
$$


Thus, if

$$
H_{t, t_{0}}:=\left\{z \in C\left(\left[t-t_{0}, t\right] ; E\right) ; z\left(t-t_{0}\right) \in \Lambda,|z(t)|_{E} \geqslant \eta\right\}
$$

we immediately have that if $x \in \Lambda$ then $z_{t-t_{0}}^{x}(0) \notin H_{t, t_{0}}$. As $H_{t, t_{0}}$ is closed, this implies that

$$
\beta:=\inf \left\{I_{t-t_{0}, t}(z) ; z \in H_{t, t_{0}}\right\}>0 .
$$

In fact, if $\beta=0$ there exists $\left\{z_{n}\right\} \subset H_{t, t_{0}}$ such that $I_{t-t_{0}, t}\left(z_{n}\right) \leqslant 1 / n$, for any $n \in \mathbb{N}$. This means that $\left\{z_{n}\right\} \subset$ $K_{\Lambda, t-t_{0}, t}(1)$ and then, as $K_{\Lambda, t-t_{0}, t}(1)$ is compact, there exists $\left\{z_{n_{k}}\right\} \subseteq\left\{z_{n}\right\}$ converging to some $\hat{z} \in K_{\Lambda, t-t_{0}, t}(1)$ in $C\left(\left[t-t_{0}, t\right] ; E\right)$. In particular, as $I_{t-t_{0}, t}$ is lower semi-continuous, we have that $I_{t-t_{0}, t}(\hat{z})=0$ and then $\hat{z}=$ $z_{t-t_{0}}^{\hat{z}\left(t-t_{0}\right)}(0)$. But this leads to a contradictions because on one side $\hat{z} \notin H_{t, t_{0}}$ (notice that $\hat{z}\left(t-t_{0}\right) \in \Lambda$ ) and on the other side $\hat{z} \in H_{t, t_{0}}$, as $H_{t, t_{0}}$ is closed.

The key point in the proof of compactness of the level sets of $V$ is given by the following result.

Proposition 5.4. Assume Hypotheses $1-5$ and assume that condition (2.14) holds. Then for any $x \in E$

$$
V(x)=\min \left\{I_{-\infty}(z) ; z \in C((-\infty, 0] ; E), \quad z(0)=x, \lim _{t \rightarrow-\infty}|z(t)|_{E}=0\right\} .
$$

Proof. Let $T>0$ and let $z \in C([0, T] ; E)$, with $z(0)=0$ and $z(T)=x$. We define

$$
\bar{z}(t):= \begin{cases}z(t+T) & \text { if } t \in[-T, 0] \\ 0 & \text { if } t \leqslant-T\end{cases}
$$

Clearly $\bar{z} \in C((-\infty, 0] ; E), \bar{z}(0)=x$ and $|\bar{z}(t)|_{E} \rightarrow 0$, as $t \rightarrow-\infty$. Moreover, $I_{-\infty}(\bar{z})=I_{-T}(\bar{z})=I_{T}(z)$ and then

$$
\min \left\{I_{-\infty}(z) ; z \in C((-\infty, 0] ; E), z(0)=x, \lim _{t \rightarrow-\infty}|z(t)|_{E}=0\right\} \leqslant I_{T}(z) .
$$

Since $T$ and $z$ are arbitrary, we get

$$
V(x) \geqslant \min \left\{I_{-\infty}(z) ; z \in C((-\infty, 0] ; E), z(0)=x, \lim _{t \rightarrow-\infty}|z(t)|_{E}=0\right\} .
$$

Thus, in order to conclude we have to prove the opposite inequality. If

$$
\min \left\{I_{-\infty}(z) ; z \in C((-\infty, 0] ; E), z(0)=x, \lim _{t \rightarrow-\infty}|z(t)|_{E}=0\right\}=\infty,
$$

there is nothing to prove. Hence, we can assume that such a minimum is finite. In Proposition 5.1 we have proved that for any $r \geqslant 0$ the level set $K_{-\infty}(r)$ is compact, so that the minimum is in fact attained by some $z_{0} \in C((-\infty, 0] ; E)$.

In fact, such a minimum $z_{0}$ is more regular. Namely we have $z_{0}(t) \in D\left((-A)^{\delta+1 / 2}\right)$, for any $t \leqslant 0$, and

$$
\lim _{t \rightarrow-\infty}\left|z_{0}(t)\right|_{D\left((-A)^{\delta+1 / 2)}\right.}=0 .
$$

Indeed, if $z_{0} \in \mathcal{K}_{-\infty}(r)$, there exists some $\varphi \in L^{2}(-\infty, 0 ; H)$, with $|\varphi|_{L^{2}(-\infty, 0 ; H)}^{2} \leqslant 3 r$, such that for any $T>0$ and $-T<t \leqslant 0$

$$
z_{0}(t)=e^{(t+T) A} z_{0}(-T)+\int_{-T}^{t} e^{(t-s) A} F\left(z_{0}(s)\right) d s+\int_{-T}^{t} e^{(t-s) A} G\left(z_{0}(s)\right) Q \varphi(s) d s .
$$

Since $\left|z_{0}(t)\right|_{E}$ is bounded for $t \in(-\infty, 0]$ (in fact it converges to zero as $t$ goes to $\left.-\infty\right)$, due to (2.7) we can take the limit above as $T$ goes to $+\infty$ and we get the following representation for $z_{0}(t)$

$$
z_{0}(t)=\int_{-\infty}^{t} e^{(t-s) A} F\left(z_{0}(s)\right) d s+\int_{-\infty}^{t} e^{(t-s) A} G\left(z_{0}(s)\right) Q \varphi(s) d s .
$$


Thanks to Lemma 3.5 this gives (5.6).

Now, according to Theorem 4.2 there exist $T_{0}>0$ and two neighborhoods $U_{1}$ and $U_{2}$ of 0 in $D\left((-A)^{\delta+1 / 2}\right)$ such that the mapping $\Gamma_{T_{0}}$ defined by

$$
\Gamma_{T_{0}} x=\int_{0}^{T_{0}} e^{\left(T_{0}-s\right) A} F\left(z^{x}(s)\right) d s+\int_{0}^{T_{0}} e^{\left(T_{0}-s\right) A} G\left(z^{x}(s)\right) Q S_{T_{0}} x d s
$$

is an homeomorphism from $U_{1}$ onto $U_{2}$. Thus, for any $\varepsilon>0$ there exists $\delta_{\varepsilon}>0$ such that

$$
|x|_{D\left((-A)^{\delta+1 / 2}\right)} \leqslant \delta_{\varepsilon} \Rightarrow\left|\Gamma_{T_{0}}^{-1} x\right|_{D\left((-A)^{\delta+1 / 2}\right)} \leqslant c_{T_{0}} \sqrt{2 \varepsilon}
$$

where $c_{T_{0}}$ is the positive constant introduced in (4.4), corresponding to $T=T_{0}$. According to the definition of $S_{T_{0}}$, $\Gamma_{T_{0}}$ and $V$ this means that

$$
|x|_{D\left((-A)^{\delta+1 / 2}\right)} \leqslant \delta_{\varepsilon} \Rightarrow\left|S_{T_{0}}\left(\Gamma_{T_{0}}^{-1} x\right)\right|_{L^{2}\left(0, T_{0} ; H\right)} \leqslant \sqrt{2 \varepsilon} \Rightarrow V(x) \leqslant \varepsilon .
$$

Now, since $z_{0}$ converges to zero in $D\left((-A)^{\delta+1 / 2}\right)$, as $t$ goes to $-\infty$, (see (5.6)) we can fix $T_{\varepsilon}>0$ such that

$$
\left|z_{0}\left(-T_{\varepsilon}\right)\right|_{D\left((-A)^{\delta+1 / 2}\right)} \leqslant \delta_{\varepsilon} \text {. }
$$

If we define

$$
z_{\varepsilon}(t):=z_{0}\left(t-\left(T_{\varepsilon}+T_{0}\right)\right), \quad t \in\left[T_{0}, T_{\varepsilon}+T_{0}\right],
$$

we have $z_{\varepsilon}\left(T_{\varepsilon}+T_{0}\right)=z_{0}(0)=x$. Moreover, $z_{\varepsilon}\left(T_{0}\right)=z_{0}\left(-T_{\varepsilon}\right)$ and then, due to (5.7) and (5.8), there exists $\varphi_{\varepsilon} \in L^{2}\left(0, T_{0} ; H\right)$ such that $z\left(\varphi_{\varepsilon}\right)(0)=0, z\left(\varphi_{\varepsilon}\right)\left(T_{0}\right)=z_{\varepsilon}\left(T_{0}\right)$ and

$$
\frac{1}{2}\left|\varphi_{\varepsilon}\right|_{L^{2}\left(0, T_{0} ; H\right)}^{2} \leqslant \varepsilon
$$

and hence $I_{T_{0}}\left(z\left(\varphi_{\varepsilon}\right)\right) \leqslant \varepsilon$. This means that if we set $z_{\varepsilon}(t):=z\left(\varphi_{\varepsilon}\right)(t), t \in\left[0, T_{0}\right]$, we have

$$
I_{T_{\varepsilon}+T_{0}}\left(z_{\varepsilon}\right) \leqslant I_{T_{0}}\left(z\left(\varphi_{\varepsilon}\right)\right)+I_{T_{0}, T_{\varepsilon}+T_{0}}\left(z_{\varepsilon}\right) \leqslant \varepsilon+I_{-T_{\varepsilon}}\left(z_{0}\right) \leqslant \varepsilon+I_{-\infty}\left(z_{0}\right) .
$$

Therefore, since $z_{\varepsilon}(0)=0$ and $z_{\varepsilon}\left(T_{\varepsilon}+T_{0}\right)=x$ we have

$$
V(x) \leqslant I_{T_{\varepsilon}+T_{0}}\left(z_{\varepsilon}\right) \leqslant \varepsilon+I_{-\infty}\left(z_{0}\right),
$$

and from the arbitrariness of $\varepsilon$ we can conclude that

$$
V(x) \leqslant I_{-\infty}\left(z_{0}\right)=\min \left\{I_{-\infty}(z) ; z \in C((-\infty, 0] ; E), z(0)=x, \lim _{t \rightarrow-\infty}|z(t)|_{E}=0\right\} .
$$

The characterization of $V$ given in Proposition 5.4 allows us to prove the compactness of the level sets of $V$.

Theorem 5.5. Under Hypotheses $1-5$, for any $r \geqslant 0$ the level set

$$
K(r):=\{x \in E: V(x) \leqslant r\}
$$

is compact in $E$.

Proof. Due to (5.3), if $G(0)=0$ the theorem is trivially true, as $K(r)=\{0\}$, for any $r \geqslant 0$. Thus, according to Hypothesis 5 we can assume that (2.14) holds.

Let $\left\{x_{n}\right\} \subset K(r)$. Thanks to Proposition 5.4, for each $n \in \mathbb{N}$ we can find $z_{n} \in C((-\infty, 0] ; E)$ with $z_{n}(0)=$ $x_{n}$ and $\left|z_{n}(t)\right|_{E}$ converging to zero, as $t$ goes to $-\infty$, such that $V\left(x_{n}\right)=I_{-\infty}\left(z_{n}\right)$. Since $V\left(x_{n}\right) \leqslant r$, we have that $\left\{z_{n}\right\} \subset K_{-\infty}(r)$. In Proposition 5.1 we have shown that $K_{-\infty}(r)$ is compact and then there exists $\left\{z_{n_{k}}\right\} \subseteq$ $\left\{z_{n}\right\}$ converging in $C((-\infty, 0] ; E)$ to some $\bar{z} \in K_{-\infty}(r)$. In particular, $x_{n_{k}}=z_{n_{k}}(0) \rightarrow \bar{z}(0)$ in $E$. Now, due to Proposition 5.4 we have $V(\bar{z}(0)) \leqslant I_{-\infty}(\bar{z}) \leqslant r$ and then $\bar{z}(0) \in K(r)$. 


\section{Lower bounds}

Theorem 6.1. For any $\delta, \gamma>0$ and $\bar{x} \in E$ there exists $\varepsilon_{0}>0$ such that

$$
\nu_{\varepsilon}\left(\left\{x \in E:|x-\bar{x}|_{E}<\delta\right\}\right) \geqslant \exp \left(-\frac{V(\bar{x})+\gamma}{\varepsilon^{2}}\right), \quad \varepsilon \leqslant \varepsilon_{0} .
$$

Proof. If $V(\bar{x})=+\infty$ there is nothing to prove. If $V(\bar{x})<+\infty$, then there exists $\bar{T}>0$ and $\bar{z} \in C([0, \bar{T}]$; $E)$ such that $\bar{z}(0)=0, \bar{z}(\bar{T})=\bar{x}$ and $\bar{z}=z(\bar{\varphi})$, for some $\bar{\varphi} \in L^{2}(0, \bar{T} ; H)$ with

$$
\frac{1}{2}|\bar{\varphi}|_{L^{2}(0, \bar{T} ; H)}^{2} \leqslant V(\bar{x})+\frac{\gamma}{2} .
$$

For such $\bar{T}$ and $\bar{x}$ we have the following crucial lemma which will be proved at the end of this section.

Lemma 6.2. For any $\delta>0$ and $R>0$ there exist $T_{0}>0$ and $\varphi_{0} \in L^{2}\left(0, \bar{T}+T_{0} ; H\right)$ such that

$$
\frac{1}{2}\left|\varphi_{0}\right|_{L^{2}\left(0, \bar{T}+T_{0} ; H\right)}^{2} \leqslant V(\bar{x})+\frac{\gamma}{2}
$$

and

$$
\sup _{|x|_{E} \leqslant R}\left|z_{0}^{x}\left(\varphi_{0}\right)\left(\bar{T}+T_{0}\right)-\bar{x}\right|_{E} \leqslant \frac{\delta}{2}
$$

According to this result, for any $x \in E$, with $|x|_{E} \leqslant R$, we have

$$
\begin{aligned}
\left|u_{\varepsilon}^{x}\left(T_{0}+\bar{T}\right)-\bar{x}\right|_{E} & \leqslant\left|u_{\varepsilon}^{x}\left(T_{0}+\bar{T}\right)-z_{0}^{x}\left(\varphi_{0}\right)\left(T_{0}+\bar{T}\right)\right|_{E}+\left|z_{0}^{x}\left(\varphi_{0}\right)\left(T_{0}+\bar{T}\right)-\bar{x}\right|_{E} \\
& \leqslant\left|u_{\varepsilon}^{x}\left(T_{0}+\bar{T}\right)-z_{0}^{x}\left(\varphi_{0}\right)\left(T_{0}+\bar{T}\right)\right|_{E}+\frac{\delta}{2},
\end{aligned}
$$

and then, due to the invariance of $v_{\varepsilon}$

$$
\begin{aligned}
v_{\varepsilon}\left(\left\{x \in E ;|x-\bar{x}|_{E}<\delta\right\}\right) & =\int_{E} \mathbb{P}\left(\left|u_{\varepsilon}^{x}\left(T_{0}+\bar{T}\right)-\bar{x}\right|_{E}<\delta\right) v_{\varepsilon}(d x) \\
& \geqslant \int_{E} \mathbb{P}\left(\left|u_{\varepsilon}^{x}\left(T_{0}+\bar{T}\right)-z_{0}^{x}\left(\varphi_{0}\right)\left(T_{0}+\bar{T}\right)\right|_{E}<\frac{\delta}{2}\right) v_{\varepsilon}(d x) \\
& \geqslant \int_{E} \mathbb{P}\left(\left|u_{\varepsilon}^{x}-z_{0}^{x}\left(\varphi_{0}\right)\right|_{C\left(\left[0, T_{0}+\bar{T}\right] ; E\right)}<\frac{\delta}{2}\right) v_{\varepsilon}(d x) .
\end{aligned}
$$

Now, as proved in [3, Theorem 6.2] for any $R>0$ there exists $\varepsilon_{0}>0$ such that for any $\varepsilon \leqslant \varepsilon_{0}$ and $|x|_{E} \leqslant R$

$$
\mathbb{P}\left(\left|u_{\varepsilon}^{x}-z_{0}^{x}\left(\varphi_{0}\right)\right|_{C\left(\left[0, T_{1}+\bar{T}\right] ; E\right)}<\frac{\delta}{2}\right) \geqslant \exp \left(-\frac{\left|\varphi_{0}\right|_{L^{2}\left(0, T_{0}+\bar{T} ; H\right)}^{2}+\gamma}{2 \varepsilon^{2}}\right) \geqslant \exp \left(-\frac{V(\bar{x})+\gamma}{\varepsilon^{2}}\right),
$$

so that

$$
v_{\varepsilon}\left(\left\{x \in E ;|x-\bar{x}|_{E}<\delta\right\}\right) \geqslant v_{\varepsilon}\left(|x|_{E} \leqslant R\right) \exp \left(-\frac{V(\bar{x})+\gamma}{\varepsilon^{2}}\right) .
$$

Therefore, we complete the proof of the theorem, if we show that there exists $\bar{R}>0$ such that

$$
\lim _{\varepsilon \rightarrow 0} v_{\varepsilon}\left(|x|_{E} \leqslant \bar{R}\right)=1 \text {. }
$$


We recall that we have taken $v_{\varepsilon}$ as the weak limit of the sequence of measures $\left\{v_{\varepsilon, n}\right\}_{n}$ defined by

$$
v_{\varepsilon, n}(\Gamma):=\frac{1}{t_{n}} \int_{0}^{t_{n}} \mathbb{P}\left(u_{\varepsilon}^{0}(s) \in \Gamma\right) d s, \quad \Gamma \in \mathcal{B}(E),
$$

for some $t_{n} \uparrow \infty$. Thus, if we show that there exists some $\bar{R}>0$ such that

$$
\lim _{\varepsilon \rightarrow 0} \sup _{s \geqslant 0} \mathbb{P}\left(\left|u_{\varepsilon}^{0}(s)\right|_{E}>\bar{R}\right)=0,
$$

we are done.

Proceeding as in [2, Proof of Proposition 6.1] we have

$$
\left|u_{\varepsilon}^{0}(t)\right|_{E} \leqslant \bar{c}\left(1+\varepsilon \sup _{s \leqslant t}\left|\gamma\left(u_{\varepsilon}^{0}\right)(s)\right|_{E}\right)
$$

where

$$
\gamma\left(u_{\varepsilon}^{0}\right)(s):=\int_{0}^{s} e^{(s-r) A} G\left(u_{\varepsilon}^{0}(r)\right) Q d w(r) .
$$

Due to [2, Propositions 4.5 and 6.1 and (4.14)], for any $\varepsilon \leqslant 1$ we have

$$
\mathbb{E} \sup _{t \geqslant 0}\left|\gamma\left(u_{\varepsilon}^{0}\right)(t)\right|_{E} \leqslant c\left(1+\mathbb{E} \sup _{t \geqslant 0}\left|u_{\varepsilon}^{0}(t)\right|_{E}\right)<\infty .
$$

Then, thanks to (6.6) for any $R>\bar{c}$

$$
\mathbb{P}\left(\left|u_{\varepsilon}^{0}(t)\right|_{E}>R\right) \leqslant \mathbb{P}\left(\sup _{s \leqslant t}\left|\gamma\left(u_{\varepsilon}^{0}\right)(t)\right|_{E}>\frac{R-\bar{c}}{\varepsilon \bar{c}}\right) \leqslant \frac{\varepsilon \bar{c}}{R-\bar{c}} c\left(1+\mathbb{E} \sup _{t \geqslant 0}\left|u_{\varepsilon}^{0}(t)\right|_{E}\right),
$$

which implies (6.5).

Now, in order to complete the proof for the lower bounds, we have to prove the lemma above.

Proof of Lemma 6.2. Let $\bar{\varphi}$ be the function introduced in (6.1). For $T>0$ fixed (to be chosen later) we define

$$
\varphi_{0}(t):= \begin{cases}0 & \text { if } t \in[0, T] \\ \bar{\varphi}(t-T) & \text { if } t \in[T, T+\bar{T}] .\end{cases}
$$

Thus, we have

$$
\left|\varphi_{0}\right|_{L^{2}(0, \bar{T}+T ; H)}^{2}=\int_{T}^{T+\bar{T}}|\bar{\varphi}(s-T)|_{H}^{2} d s=|\bar{\varphi}|_{L^{2}(0, \bar{T} ; H)}^{2}
$$

and due to (6.1) we obtain (6.2).

Now, for any $x \in E$ we consider $z_{0}^{x}\left(\varphi_{0}\right)$, the solution of the skeleton equation (3.2) starting from $x$ at time zero and corresponding to the control $\varphi_{0}$. Due to the definition of $\varphi_{0}$, it is immediate to check that

$$
z_{0}^{x}\left(\varphi_{0}\right)(t)= \begin{cases}z_{0}^{x}(0)(t) & \text { if } t \in[0, T], \\ z_{0}^{z_{0}^{x}(0)(T)}\left(\varphi_{0}\right)(t) & \text { if } t \in[T, T+\bar{T}],\end{cases}
$$

where $z_{0}^{x}(0)$ is the solution of the uncontrolled problem (3.26) starting from $x$ at time zero and $z_{T}^{z_{0}^{x}(0)(T)}\left(\varphi_{0}\right)$ is the solution of the skeleton equation (3.2) starting from $z_{0}^{x}(0)(T)$ at time $T$ with control $\varphi_{0}$. 
If we set $\psi(t):=z_{0}^{x}\left(\varphi_{0}\right)(t+T), t>0$, with a simple change of variable we have

$$
\begin{aligned}
\psi(t) & =e^{t A} z_{0}^{x}(0)(T)+\int_{T}^{T+t} e^{(T+t-s) A} F\left(z_{0}^{x}\left(\varphi_{0}\right)(s)\right) d s+\int_{T}^{T+t} e^{(T+t-s) A} G\left(z_{0}^{x}\left(\varphi_{0}\right)(s)\right) Q \bar{\varphi}(s-T) d s \\
& =e^{t A} z_{0}^{x}(0)(T)+\int_{0}^{t} e^{(t-s) A} F\left(z_{0}^{x}\left(\varphi_{0}\right)(s+T)\right) d s+\int_{0}^{t} e^{(t-s) A} G\left(z_{0}^{x}\left(\varphi_{0}\right)(s+T)\right) Q \bar{\varphi}(s) d s,
\end{aligned}
$$

so that for any $t \in[0, \bar{T}]$

$$
\begin{aligned}
\psi(t)-z_{0}^{0}(\bar{\varphi})(t)= & e^{t A} z_{0}^{x}(0)(T)+\int_{0}^{t} e^{(t-s) A}\left[F(\psi(s))-F\left(z_{0}^{0}(\bar{\varphi})(s)\right)\right] d s \\
& +\int_{0}^{t} e^{(t-s) A}\left[G(\psi(s))-G\left(z_{0}^{0}(\bar{\varphi})(s)\right)\right] Q \bar{\varphi}(s) d s .
\end{aligned}
$$

Recalling that $\left|e^{t A} x\right|_{E} \leqslant e^{-\alpha t}|x|_{E}$, for any $x \in E$ and $t \geqslant 0$, we have

$$
\begin{aligned}
\left|\psi(t)-z_{0}^{0}(\bar{\varphi})(t)\right|_{E} \leqslant & e^{-\alpha t}\left|z_{0}^{x}(0)(T)\right|_{E}+\int_{0}^{t} e^{-\alpha(t-s)}\left|F(\psi(s))-F\left(z_{0}^{0}(\bar{\varphi})(s)\right)\right|_{E} d s \\
& +\left|\int_{0}^{t} e^{(t-s) A}\left[G(\psi(s))-G\left(z_{0}^{0}(\bar{\varphi})(s)\right)\right] Q \bar{\varphi}(s) d s\right|_{E}
\end{aligned}
$$

By proceeding as in [2, proof of Theorem 4.2] (where stochastic convolutions are studied), for any $t \in[0, \bar{T}]$ and $p$ large enough we have

$$
\begin{aligned}
& \left|\int_{0}^{t} e^{(t-s) A}\left[G(\psi(s))-G\left(z_{0}^{0}(\bar{\varphi})(s)\right)\right] Q \bar{\varphi}(s) d s\right|_{E} \\
& \quad \leqslant e^{-\alpha t}\|Q\|_{\rho}|\bar{\varphi}|_{L^{2}(0, \bar{T} ; H)} c_{p}(\bar{T})\left(\int_{0}^{t} f_{p}(s)\left(\sup _{\sigma \leqslant s} e^{\alpha \sigma}\left|\psi(\sigma)-z_{0}^{0}(\bar{\varphi})(\sigma)\right|_{E}\right)^{p} d s\right)^{\frac{1}{p}},
\end{aligned}
$$

where $\|Q\|_{\rho}$ is defined in (2.10) and

$$
f_{p}(s):=\left(\int_{0}^{s}(s-\sigma)^{-\eta} d \sigma\right)^{\frac{p}{2}}
$$

for some positive constant $\eta$ less that 1 specified in [2, Proof of Theorem 4.2].

Moreover, as $\psi(t)=z_{0}^{x}\left(\varphi_{0}\right)(t+T), t \geqslant 0$, due to (3.3) we have

$$
\sup _{t \geqslant 0}|\psi(t)|_{E} \leqslant c_{\left|\varphi_{0}\right|_{L^{2}(0, T+\bar{T} ; H)}}\left(1+|x|_{E}\right)=c_{|\bar{\varphi}|_{L^{2}(0, \bar{T} ; H)}}\left(1+|x|_{E}\right)
$$

and analogously

$$
\sup _{t \geqslant 0}\left|z_{0}^{0}(\bar{\varphi})(t)\right|_{E} \leqslant c_{|\bar{\varphi}|_{L^{2}(0, \bar{T} ; H)}} .
$$


As $F$ is locally Lipschitz-continuous, this implies that there exists $L_{R}>0$ (depending also on $|\bar{\varphi}|_{L^{2}(0, \bar{T} ; H)}$ ) such that if $|x|_{E} \leqslant R$

$$
\begin{aligned}
\int_{0}^{t} e^{-\alpha(t-s)}\left|F(\psi(s))-F\left(z_{0}^{0}(\bar{\varphi})(s)\right)\right|_{E} d s & \leqslant L_{R} e^{-\alpha t} \int_{0}^{t} e^{\alpha s}\left|\psi(s)-z_{0}^{0}(\bar{\varphi})(s)\right|_{E} d s \\
& \leqslant L_{R} e^{-\alpha t} \int_{0}^{t} \sup _{\sigma \leqslant s} e^{\alpha \sigma}\left|\psi(\sigma)-z_{0}^{0}(\bar{\varphi})(\sigma)\right|_{E} d s .
\end{aligned}
$$

Hence, collecting all terms we obtain

$$
\begin{aligned}
e^{\alpha t}\left|\psi(t)-z_{0}^{0}(\bar{\varphi})(t)\right|_{E} \leqslant & \left|z_{0}^{x}(0)(T)\right|_{E}+L_{R} \int_{0}^{t} \sup _{\sigma \leqslant s} e^{\alpha \sigma}\left|\psi(\sigma)-z_{0}^{0}(\bar{\varphi})(\sigma)\right|_{E} d s \\
& +\tilde{c}_{p}(\bar{T})\left(\int_{0}^{t} f_{p}(s) \sup _{\sigma \leqslant s} e^{\alpha p \sigma}\left|\psi(\sigma)-z_{0}^{0}(\bar{\varphi})(\sigma)\right|_{E}^{p} d s\right)^{\frac{1}{p}},
\end{aligned}
$$

so that

$$
\begin{aligned}
\sup _{s \leqslant t} e^{\alpha p s}\left|\psi(s)-z_{0}^{0}(\bar{\varphi})(s)\right|_{E}^{p} \leqslant & 3^{p}\left|z_{0}^{x}(0)(T)\right|_{E}^{p}+3^{p} L_{R}^{p} \bar{T}^{p-1} \int_{0}^{s} \sup _{\sigma \leqslant r} e^{\alpha p \sigma}\left|\psi(\sigma)-z_{0}^{0}(\bar{\varphi})(\sigma)\right|_{E}^{p} d r \\
& +3^{p} \tilde{c}_{p}(\bar{T})^{p} \int_{0}^{t} f_{p}(s) \sup _{\sigma \leqslant s} e^{\alpha p \sigma}\left|\psi(\sigma)-z_{0}^{0}(\bar{\varphi})(\sigma)\right|_{E}^{p} d s .
\end{aligned}
$$

Therefore, by using the Gronwall lemma we obtain

$$
\sup _{s \leqslant t} e^{\alpha p s}\left|\psi(s)-z_{0}^{0}(\bar{\varphi})(s)\right|_{E}^{p} \leqslant 3^{p}\left|z_{0}^{x}(0)(T)\right|_{E}^{p} \exp \left(\int_{0}^{t} g(s) d s\right),
$$

where

$$
g(s):=3^{p} L_{R}^{p} \bar{T}^{p-1}+3^{p} \tilde{c}_{p}(\bar{T})^{p} f_{p}(s) .
$$

This yields

$$
\left|\psi(\bar{T})-z_{0}^{0}(\bar{\varphi})(\bar{T})\right|_{E} \leqslant e^{-\alpha \bar{T}} c_{p}\left(\bar{T}, R,|\bar{\varphi}|_{L^{2}(0, \bar{T} ; H)}\right)\left|z_{0}^{x}(0)(T)\right|_{E},
$$

that is

$$
\left|z_{0}^{x}\left(\varphi_{0}\right)(T+\bar{T})-\bar{x}\right|_{E} \leqslant c_{p}\left(\bar{T}, R,|\bar{\varphi}|_{L^{2}(0, \bar{T})}\right)\left|z_{0}^{x}(0)(T)\right|_{E} .
$$

According to (3.27)

$$
\lim _{T \rightarrow \infty} \sup _{|x|_{E} \leqslant R}\left|z_{0}^{x}(0)(T)\right|_{E}=0,
$$

and then we can find $T_{0}>0$ such that

$$
\left|z_{0}^{x}\left(\varphi_{0}\right)\left(T_{0}+\bar{T}\right)-\bar{x}\right|_{E} \leqslant \frac{\delta}{2} .
$$




\section{Upper bounds}

Before proceeding with the proof of upper bounds we need the following preliminary result.

Lemma 7.1. Under Hypotheses $1-5$, for any $\delta, s>0$ there exist $\lambda>0$ and $\bar{T}>0$ such that

$$
\left\{z(t) ; z \in K_{\Sigma_{\lambda}, t}(s)\right\} \subseteq\left\{x \in E ; \operatorname{dist}_{E}(x, K(s))<\frac{\delta}{2}\right\}, \quad t \geqslant \bar{T},
$$

where $\Sigma_{\lambda}:=\left\{x \in E ;|x|_{E} \leqslant \lambda\right\}$.

Proof. If this is not true, there exist two sequences $\left\{\lambda_{n}\right\} \downarrow 0$ and $\left\{T_{n}\right\} \uparrow+\infty$ and $z_{n} \in K_{\Sigma_{\lambda_{n}}, T_{n}}(s)$ such that

$$
\operatorname{dist}_{E}\left(z_{n}\left(T_{n}\right), K(s)\right) \geqslant \frac{\delta}{2}, \quad n \in \mathbb{N}
$$

Thus, if we define $\bar{z}_{n}(t):=z_{n}\left(t+T_{n}\right), t \in\left[-T_{n}, 0\right]$, for each $n \in \mathbb{N}$ we have $\bar{z}_{n}(0)=z_{n}\left(T_{n}\right)$ and

$$
\operatorname{dist}_{E}\left(\bar{z}_{n}(0), K(s)\right) \geqslant \frac{\delta}{2}, \quad n \in \mathbb{N} .
$$

Moreover, since $\lambda_{n} \downarrow 0$ and $T_{n} \uparrow+\infty$, for any $k \in \mathbb{N}$ we have $\left\{z_{n}\right\}_{n \geqslant k} \subset K_{\Sigma_{\lambda_{k}}, T_{k}}(s)$.

Now, for any $t \geqslant-T_{n}$ we have

$$
\bar{z}_{n}(t)=z_{n}\left(t+T_{n}\right)=e^{\left(t+T_{n}\right) A} z_{n}(0)+\int_{0}^{t+T_{n}} e^{\left(t+T_{n}-s\right) A} F\left(z_{n}(s)\right) d s+\gamma_{\varphi_{n}}^{0}\left(z_{n}\right)\left(t+T_{n}\right),
$$

where $\varphi_{n}$ is some function in $L^{2}\left(0, T_{n} ; H\right)$, with $\left|\varphi_{n}\right|_{L^{2}\left(0, T_{n} ; H\right)}^{2} \leqslant 3 s$, and $\gamma_{\varphi_{n}}^{0}\left(z_{n}\right)$ is defined as in the proof of Theorem 3.1. Thus, with the same notations as in Section 3 we have

$$
\bar{z}_{n}(t)=z_{0}^{z_{n}(0)}\left(\varphi_{n}\right)\left(t+T_{n}\right), \quad t \geqslant-T_{n}
$$

and then, thanks to (3.3), we have

$$
\sup _{t \in\left[-T_{n}, 0\right]}\left|\bar{z}_{n}(t)\right|_{E} \leqslant c_{S}\left(1+\lambda_{1}\right), \quad n \geqslant 1 .
$$

Moreover, thanks to (3.4) for any $t>-T_{n}$ we have

$$
\left|\bar{z}_{n}(t)\right|_{C_{\star}^{\theta_{\star}\left(\overline{\mathcal{O}} ; \mathbb{R}^{r}\right)}} \leqslant c_{S}\left(1+\left|z_{n}(0)\right|_{E}^{m}\right)\left(1+\left(t+T_{n}\right)^{-\frac{\theta_{\star}}{2}}\right),
$$

so that, for any $n \geqslant k$ and $t \geqslant-T_{k} / 2$

$$
\left|\bar{z}_{n}(t)\right|_{C^{\theta_{\star}\left(\overline{\mathcal{O}} ; \mathbb{R}^{r}\right)}} \leqslant c_{s}\left(1+\lambda_{k}^{m}\right)\left(1+\left(T_{k} / 2 \wedge 1\right)^{-\frac{\theta_{\star}}{2}}\right):=\rho_{k} .
$$

In particular for any $k \in \mathbb{N}$

$$
\bar{z}_{n_{\mid\left[-T_{k} / 2,0\right]}} \in K_{\Lambda_{\rho_{k}},-T_{k} / 2,0}(s), \quad n \geqslant k,
$$

where

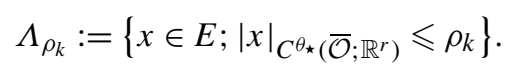

In Section 5 (see also [3]) we have seen that for each $k \in \mathbb{N}$ the set $K_{\Lambda_{\rho_{k}},-T_{k} / 2,0}(s)$ is compact in $C\left(\left[-T_{k} / 2,0\right] ; E\right)$. Thus there exist $\left\{\bar{z}_{n_{1}}\right\} \subseteq\left\{\bar{z}_{n}\right\}$ and $\hat{z}_{1} \in K_{\Lambda_{\rho_{1}},-T_{1} / 2,0}(s)$ such that $\bar{z}_{n_{1}}$ converges to $\hat{z}_{1}$ in $C\left(\left[-T_{1} / 2,0\right] ; E\right)$. Analogously, there exist $\left\{\bar{z}_{n_{2}}\right\} \subseteq\left\{\bar{z}_{n_{1}}\right\}$ and $\hat{z}_{2} \in K_{\Lambda_{\rho_{2}},-T_{2} / 2,0}(s)$ such that $\bar{z}_{n_{2}}$ converges to $\hat{z}_{2}$ in $C\left(\left[-T_{2} / 2,0\right] ; E\right)$. 
Moreover $\hat{z}_{2} \equiv \hat{z}_{1}$ on $\left[-T_{1} / 2,0\right]$. By proceeding in this way we find a subsequence $\left\{\bar{z}_{n^{\prime}}\right\} \subseteq\left\{\bar{z}_{n}\right\}$ converging in $C((-\infty, 0 ; E)$ to some $\hat{z}$ such that

$$
I_{-\infty}(\hat{z}) \leqslant s, \quad \sup _{t \leqslant 0}|\hat{z}(t)|_{E} \leqslant c\left(1+\lambda_{1}\right) .
$$

This means that we can apply Lemma 5.2 and obtain that $|\hat{z}(t)|_{C_{\star}^{\theta_{\star}}\left(\overline{\mathcal{O}} ; \mathbb{R}^{r}\right)} \leqslant L(s)$, for some constant $L(s)>0$. Then, by proceeding as in the proof of Proposition 5.1 we can conclude that $|\hat{z}(t)|_{E} \rightarrow 0$, as $t \rightarrow-\infty$, and hence $\hat{z} \in K_{-\infty}(s)$. According to Proposition 5.4 this implies that $V(\hat{z}(0)) \leqslant I_{-\infty}(\hat{z}) \leqslant s$. But this is not possible, as

$$
\operatorname{dist}_{E}(\hat{z}(0), K(s)) \geqslant \frac{\delta}{2} \text {. }
$$

In the proof of upper bounds we distinguish the two cases of bounded and unbounded $G$.

\subsection{Upper bounds when $G$ is bounded}

In [3, Theorem 3.3] we have proved that if

$$
\sup _{(\xi, \sigma) \overline{\mathcal{O}} \times \mathbb{R}^{r}}|g(\xi, \sigma)|_{\mathcal{L}\left(\mathbb{R}^{r}\right)}<\infty,
$$

then for any $T, R>0$ and $s>0$ there exists $\rho>0$ such that

$$
\sup _{|x|_{E} \leqslant R} \mathbb{P}\left(\left|u_{\varepsilon}^{x}\right|_{C([0, T] ; E)} \geqslant \rho\right) \leqslant \exp \left(-\frac{s}{\varepsilon^{2}}\right), \quad \varepsilon \in(0,1] .
$$

Here we are assuming that there exists $\alpha>0$ such that for any $x \in E$ and $h \in D(A)$

$$
\left\langle A h, \delta_{h}\right\rangle+\left\langle F(x+h)-F(x), \delta_{h}\right\rangle \leqslant-\alpha|h|_{E},
$$

for some $\delta_{h} \in \partial|h|_{E}$. Thus, by adapting the proofs of [3, Theorems 3.2 and 3.3] it is possible to show that for any $R>0$ and $s>0$ there exists $\rho>0$ such that

$$
\sup _{|x|_{E} \leqslant R} \mathbb{P}\left(\left|u_{\varepsilon}^{x}\right|_{C([0,+\infty) ; E)} \geqslant \rho\right) \leqslant \exp \left(-\frac{s}{\varepsilon^{2}}\right), \quad \varepsilon \in(0,1] .
$$

We recall that in the present paper for each $\varepsilon>0$ we have defined the measure $v_{\varepsilon}$ as the weak limit of the sequence of probability measures $\left\{v_{\varepsilon, n}\right\}_{n \geqslant 1}$ defined by

$$
v_{\varepsilon, n}(\Gamma):=\frac{1}{t_{n}} \int_{0}^{t_{n}} \mathbb{P}\left(u_{\varepsilon}^{0}(t) \in \Gamma\right) d t, \quad \Gamma \in \mathcal{B}(E),
$$

for some sequence $t_{n} \uparrow \infty$ possibly depending on $\varepsilon$. Therefore, from (7.2) we obtain that for any $s>0$ there exists $\rho>0$ such that for any $\varepsilon \in(0,1]$

$$
v_{\varepsilon}\left(\left\{x \in E ;|x|_{E}>\rho\right\}\right) \leqslant \liminf _{n \rightarrow \infty} v_{\varepsilon, n}\left(\left\{x \in E ;|x|_{E}>\rho\right\}\right) \leqslant \exp \left(-\frac{s}{\varepsilon^{2}}\right) .
$$

Now, for any $n \in \mathbb{N}$ and $\rho, s, \delta>0$ we define

$$
H_{\rho, s, \delta}(n):=\left\{z \in C([0, n] ; E) ;|z(0)|_{E} \leqslant \rho,|z(j)|_{E} \geqslant \lambda, j=1, \ldots, n\right\},
$$

where $\rho$ is any positive constant and $\lambda$ is the constant introduced in Lemma 7.1 corresponding to $s$ and $\delta$.

Lemma 7.2. Under Hypotheses $1-4$, for any $\rho, s, \delta>0$ there exists $\bar{n} \in \mathbb{N}$ such that

$$
\beta_{\bar{n}}:=\inf \left\{I_{\bar{n}}(z) ; z \in H_{\rho, s, \delta}(\bar{n})\right\}>s .
$$


Proof. If this is not true, then $\sup _{n} \beta_{n} \leqslant s$. Thus, for each $n \in \mathbb{N}$ we can fix $z_{n} \in H_{\rho, s, \delta}(n)$ such that

$$
I_{n}\left(z_{n}\right) \leqslant \inf \left\{I_{n}(z) ; z \in H_{\rho, s, \delta}(n)\right\}+1=\beta_{n}+1 \leqslant s+1 .
$$

Due to (3.4) this easily implies that

$$
\sup _{t \geqslant 1}\left|z_{n}(t)\right|_{C_{\star}^{\theta_{\star}\left(\overline{\mathcal{O}} ; \mathbb{R}^{r}\right)}} \leqslant c_{S}\left(1+\left|z_{n}(0)\right|_{E}^{m}\right) \leqslant c_{S}\left(1+\rho^{m}\right)=: c_{\rho, s} .
$$

Now, if we show that for $k \in \mathbb{N}$ large enough

$$
v_{k}:=\inf \left\{I_{k}(z) ; z \in C([0, k] ; E),|z(0)|_{E} \leqslant c_{\rho, s} \wedge \rho,|z(k)|_{E} \geqslant \lambda\right\}>0,
$$

we are led to a contradiction. Indeed, after fixing such $\bar{k}$ thanks to (7.5) we have

$$
s+1 \geqslant I_{n \bar{k}}\left(z_{n \bar{k}}\right) \geqslant n v_{\bar{k}}
$$

and this is clearly not possible, as $n$ can be taken arbitrarily large.

Hence, to finish the proof of the lemma, it remains to prove (7.6). For any $x \in E$ we consider the solution $z_{1}^{x}$ of problem (3.26) (corresponding to $T=1$ ). Due to (3.27), there exists $\bar{t} \geqslant 1$ such that

$$
|x|_{E} \leqslant c_{\rho, s} \wedge \rho \Rightarrow\left|z_{1}^{x}(t)\right|_{E} \leqslant \frac{\lambda}{2}, \quad t \geqslant \bar{t}
$$

and then, if we fix any integer $\bar{k} \geqslant \bar{t}$ we have

$$
z_{1_{[1, \bar{k}]}}^{x} \notin\left\{z \in C([1, \bar{k}] ; E) ;|z(\bar{k})|_{E} \geqslant \lambda\right\} .
$$

Our aim is to prove that for such $\bar{k}$ we have $v_{\bar{k}}>0$. If $v_{\bar{k}}=0$, then there exists

$$
\left\{\hat{z}_{n}\right\} \subset\left\{z \in C([0, \bar{k}] ; E) ;|z(0)|_{E} \leqslant c_{\rho, s} \wedge \rho,|z(\bar{k})|_{E} \geqslant \lambda\right\},
$$

such that $I_{\bar{k}}\left(\hat{z}_{n}\right)$ converges to zero, as $n \rightarrow \infty$. Hence, as $\left|\hat{z}_{n}(0)\right|_{E} \leqslant \rho$, we have $\left|\hat{z}_{n}(1)\right|_{C_{*}\left(\overline{\mathcal{O}} ; \mathbb{R}^{r}\right)} \leqslant c_{\rho, s}$, so that there exist $\left\{\hat{z}_{n_{j}}\right\} \subseteq\left\{\hat{z}_{n}\right\}$ and $\bar{x} \in E$ such that $\hat{z}_{n_{j}}(1) \rightarrow \bar{x}$. Now, since $I_{\bar{k}}\left(\hat{z}_{n}\right) \rightarrow 0$, there exists a sequence $\left\{\varphi_{n}\right\} \subset$ $L^{2}(0, \bar{k} ; H)$ converging to zero such that $\hat{z}_{n}=z\left(\varphi_{n}\right)$. This implies that $\hat{z}_{n_{j}}$ converges to $z_{1}^{\bar{x}}$ in $C([1, \bar{k}] ; E)$ and then $\left|z_{1}^{\bar{x}}(\bar{k})\right|_{E} \geqslant \lambda$. But in fact, due to (7.7), this is not possible.

With arguments analogous to those used by Sowers in [14] now we obtain the upper bounds.

Theorem 7.3. Assume that Hypotheses 1-5 hold. Moreover, assume that $g$ is uniformly bounded, that is

$$
\sup _{(\xi, \sigma) \overline{\mathcal{O}} \times \mathbb{R}^{r}}|g(\xi, \sigma)|_{\mathcal{L}\left(\mathbb{R}^{r}\right)}<\infty .
$$

Then, for any $s, \delta, \gamma>0$ there exists $\varepsilon_{0}>0$ such that

$$
v_{\varepsilon}\left(\left\{x \in E ; \operatorname{dist}_{E}(x, K(s)) \geqslant \delta\right\}\right)<\exp \left(-\frac{s-\gamma}{\varepsilon^{2}}\right), \quad \varepsilon \leqslant \varepsilon_{0} .
$$

Proof. Due to the invariance of the measure $v_{\varepsilon}$, for any $t \geqslant 0$ we have

$$
v_{\varepsilon}\left(\left\{x \in E ; \operatorname{dist}_{E}(x, K(s)) \geqslant \delta\right\}\right)=\int_{E} \mathbb{P}\left(\operatorname{dist}_{E}\left(u_{\varepsilon}^{y}(t), K(s)\right) \geqslant \delta\right) v_{\varepsilon}(d y) .
$$

Thus, according to (7.3), for $s>0$ fixed we can find $\rho>0$ such that for any $\varepsilon \in(0,1]$ 


$$
\begin{aligned}
& v_{\varepsilon}\left(\left\{x \in E ; \operatorname{dist}_{E}(x, K(s)) \geqslant \delta\right\}\right) \\
& \quad=\int_{|y|_{E}>\rho} \mathbb{P}\left(\operatorname{dist}_{E}\left(u_{\varepsilon}^{y}(t), K(s)\right) \geqslant \delta\right) v_{\varepsilon}(d y)+\int_{|y|_{E} \leqslant \rho} \mathbb{P}\left(\operatorname{dist}_{E}\left(u_{\varepsilon}^{y}(t), K(s)\right) \geqslant \delta\right) v_{\varepsilon}(d y) \\
& \quad \leqslant \exp \left(-\frac{s}{\varepsilon^{2}}\right)+\int_{|y| E \leqslant \rho} \mathbb{P}\left(\operatorname{dist}_{E}\left(u_{\varepsilon}^{y}(t), K(s)\right) \geqslant \delta\right) v_{\varepsilon}(d y) .
\end{aligned}
$$

If $H_{\rho, s, \delta}(n)$ is the closed set defined in (7.4), by using the upper bounds for the path large deviations from [3, Theorem 6.3] in its equivalent formulation due to Donsker and Varadhan (see [7] and [16]), due to Lemma 7.2 we can fix $\bar{n} \in \mathbb{N}$ and $\varepsilon_{1}>0$ such that

$$
\sup _{|y|_{E} \leqslant \rho} \mathbb{P}\left(u_{\varepsilon}^{y} \in H_{\rho, s, \delta}(\bar{n})\right) \leqslant \exp \left(-\frac{s-\gamma / 2}{\varepsilon^{2}}\right), \quad \varepsilon \leqslant \varepsilon_{1} .
$$

Thus, from (7.8) for any $\varepsilon \leqslant \varepsilon_{1}$ we obtain

$$
\begin{aligned}
v_{\varepsilon}\left(\left\{x \in E ; \operatorname{dist}_{E}(x, K(s)) \geqslant \delta\right\}\right) \leqslant & \exp \left(-\frac{s}{\varepsilon^{2}}\right)+\exp \left(-\frac{s-\gamma / 2}{\varepsilon^{2}}\right) \\
& +\int_{|y|} \mathbb{P}\left(\operatorname{dist}_{E}\left(u_{\varepsilon}^{y}(t), K(s)\right) \geqslant \delta, u_{\varepsilon}^{y} \notin H_{\rho, s, \delta}(\bar{n})\right) v_{\varepsilon}(d y) .
\end{aligned}
$$

Concerning the integral above, recalling how $\Sigma_{\lambda}$ has been defined in Lemma 7.1, we have

$$
\begin{aligned}
& \int_{|y|_{E} \leqslant \rho} \mathbb{P}\left(\operatorname{dist}_{E}\left(u_{\varepsilon}^{y}(t), K(s)\right) \geqslant \delta, u_{\varepsilon}^{y} \notin H_{\rho, s, \delta}(\bar{n})\right) \nu_{\varepsilon}(d y) \\
& \leqslant \int_{|y|_{E} \leqslant \rho} \mathbb{P}\left(\bigcup_{k=1}^{\bar{n}}\left\{\operatorname{dist}_{E}\left(u_{\varepsilon}^{y}(t), K(s)\right) \geqslant \delta, u_{\varepsilon}^{y}(k) \in \Sigma_{\lambda}\right\}\right)
\end{aligned}
$$

and then, due to the Markov property of $u_{\varepsilon}^{y}$, for any $t>\bar{n}$ we get

$$
\begin{aligned}
& \int_{|y| E \leqslant \rho} \mathbb{P}\left(\operatorname{dist}_{E}\left(u_{\varepsilon}^{y}(t), K(s)\right) \geqslant \delta, u_{\varepsilon}^{y} \notin H_{\rho, s, \delta}(\bar{n})\right) v_{\varepsilon}(d y) \\
& \leqslant \sum_{k=1}^{\bar{n}} \sup _{y \in \Sigma_{\lambda}} \mathbb{P}\left(\operatorname{dist}_{E}\left(u_{\varepsilon}^{y}(t-k), K(s)\right) \geqslant \delta\right) .
\end{aligned}
$$

Now, thanks to Lemma 7.1 there exists $\bar{T}>0$ such that for any $t \geqslant \bar{T}$ and $y \in \Sigma_{\lambda}$

$$
\mathbb{P}\left(\operatorname{dist}_{E}\left(u_{\varepsilon}^{y}(t), K(s)\right) \geqslant \delta\right) \leqslant \mathbb{P}\left(\left|u_{\varepsilon}^{y}-K_{\Sigma_{\lambda}, t}(s)\right|_{C([0, t] ; E)} \geqslant \frac{\delta}{2}\right) \leqslant \mathbb{P}\left(\left|u_{\varepsilon}^{y}-K_{y, t}(s)\right|_{C([0, t] ; E)} \geqslant \frac{\delta}{2}\right) .
$$

Thus, by using the upper bounds for the trajectories of the solution $u_{\varepsilon}^{y}$ proved in [3, Theorem 6.3], we can find $\varepsilon(t)>0$ such that

$$
\sup _{y \in \Sigma_{\lambda}} \mathbb{P}\left(\operatorname{dist}_{E}\left(u_{\varepsilon}^{y}(t), K(s)\right) \geqslant \delta\right) \leqslant \exp \left(-\frac{s-\gamma / 2}{\varepsilon^{2}}\right), \quad \varepsilon \leqslant \varepsilon(t) .
$$

This means that if we take $t:=\bar{T}+\bar{n}$ and $\varepsilon_{2}:=\min \{\varepsilon(t-k), k=1, \ldots, \bar{n}\}$, for any $\varepsilon \leqslant \varepsilon_{2}$

$$
\int_{|y|_{E} \leqslant \rho} \mathbb{P}\left(\operatorname{dist}_{E}\left(u_{\varepsilon}^{y}(t), K(s)\right) \geqslant \delta, u_{\varepsilon}^{y} \notin H_{\rho, s, \delta}(\bar{n})\right) \nu_{\varepsilon}(d y) \leqslant \bar{n} \exp \left(-\frac{s-\gamma / 2}{\varepsilon^{2}}\right) .
$$


Hence, from (7.9) and the inequality above we can conclude that for $\varepsilon \leqslant \varepsilon_{3}:=\varepsilon_{1} \wedge \varepsilon_{2} \wedge 1$

$$
v_{\varepsilon}\left(\left\{x \in E ; \operatorname{dist}_{E}(x, K(s)) \geqslant \delta\right\}\right) \leqslant \exp \left(-\frac{s}{\varepsilon^{2}}\right)+(1+\bar{n}) \exp \left(-\frac{s-\gamma / 2}{\varepsilon^{2}}\right),
$$

which yields upper bounds by taking some $\varepsilon_{0} \leqslant \varepsilon_{3}$ sufficiently small.

\subsection{Upper bounds when $G$ is unbounded}

For any $n \in \mathbb{N}$ and $s, \delta>0$ we define

$$
H_{s, \delta}(n):=\left\{z \in C([0, n] ; E) ;|z(j)|_{E} \geqslant \lambda, j=1, \ldots, n\right\},
$$

where $\lambda$ is the constant introduced in Lemma 7.1 corresponding to $s$ and $\delta$. Notice that, unlike the functions of the set $H_{\rho, s, \delta}(n)$ defined in (7.4), the functions belonging to $H_{s, \delta}(n)$ have no conditions on their initial value. This is because in the proof of the upper bounds we don't want to use the exponential estimate (7.3), where the assumption of boundedness of $G$ is needed. Nevertheless, due to the estimates of $\left|z^{x}(\varphi(t))\right|_{E}$ proved in Theorem 3.4, which are uniform with respect to the initial datum $x \in E$, we can prove a result analogous to Lemma 7.2 also in the case of unbounded $G$.

Lemma 7.4. Assume that Hypotheses $1-4$ and 6 hold. Then for any $s, \delta>0$ there exists $\bar{n} \in \mathbb{N}$ such that

$$
\beta_{\bar{n}}:=\inf \left\{I_{\bar{n}}(z) ; z \in H_{s, \delta}(\bar{n})\right\}>s .
$$

Proof. If (7.10) does not hold, we have $\sup _{n} \beta_{n} \leqslant s$ and then, as in the proof of Lemma 7.2, for each $n \in \mathbb{N}$ we can fix $z_{n} \in H_{s, \delta}(n)$ such that $I_{n}\left(z_{n}\right) \leqslant \beta_{n}+1 \leqslant s+1$. This means that $z_{n}=z_{0}^{z_{n}(0)}\left(\varphi_{n}\right)$, for some $\varphi \in L^{2}(0, n ; H)$ such that $\left|\varphi_{n}\right|_{L^{2}(0, n ; H)}^{2} \leqslant 3(s+1)$, and then, thanks to Theorem 3.4, we have

$$
\sup _{n \in \mathbb{N}}\left|z_{n}(1 / 2)\right|_{E}=: c_{s}<+\infty .
$$

According to (3.4) this yields

$$
\sup _{\substack{t \geqslant 1 \\ n \in \mathbb{N}}}\left|z_{n}(t)\right|_{C_{\star}^{\theta_{\star}}\left(\overline{\mathcal{O}} ; \mathbb{R}^{r}\right)}=: c_{s}^{\prime}<+\infty .
$$

Now we show that there exists $k \in \mathbb{N}$ such that

$$
v_{k}:=\inf \left\{I_{k}(z) ; z \in C([1, k] ; E),|z(1)|_{C^{\theta_{\star}}\left(\overline{\mathcal{O}} ; \mathbb{R}^{r}\right)} \leqslant c_{s}^{\prime},|z(k)|_{E} \geqslant \lambda\right\}>0 .
$$

If $z_{1}^{x}$ denotes the solution of the uncontrolled problem (3.26), starting from $x \in E$ at time 1, as in the proof of Lemma 7.2 we can fix $\bar{k}$ such that

$$
|x|_{C_{\star}^{\theta_{\star}}\left(\overline{\mathcal{O}} ; \mathbb{R}^{r}\right)} \leqslant c_{S}^{\prime} \Rightarrow z_{1_{[1, \bar{k}]}^{x}}^{x} \notin\left\{z \in C([1, \bar{k}] ; E) ;|z(\bar{k})|_{E} \geqslant \lambda\right\} .
$$

With the same arguments used in the proof of Lemma 7.2, we can prove that $v_{\bar{k}}>0$ for such $\bar{k}$. Thus, as in the proof of Lemma 7.2 we get a contradiction, as for $n$ arbitrarily large, due to (7.11) we obtain

$$
s+1 \geqslant I_{n \bar{k}+1}\left(z_{n \bar{k}+1}\right) \geqslant n v_{\bar{k}} .
$$

The previous lemma allows us to adapt the proof of Theorem 7.3 to the case of an unbounded diffusion term $G$.

Theorem 7.5. Assume that Hypotheses 1-6 hold. Then, for any $s, \delta, \gamma>0$ there exists $\varepsilon_{0}>0$ such that

$$
v_{\varepsilon}\left(\left\{x \in E ; \operatorname{dist}_{E}(x, K(s)) \geqslant \delta\right\}\right)<\exp \left(-\frac{s-\gamma}{\varepsilon^{2}}\right), \quad \varepsilon \leqslant \varepsilon_{0} .
$$


Proof. For any $t \geqslant 0$ we have

$$
\begin{aligned}
v_{\varepsilon}\left(\left\{x \in E, \operatorname{dist}_{E}(x, K(s)) \geqslant \delta\right\}\right)= & \int_{E} \mathbb{P}\left(\operatorname{dist}_{E}\left(u_{\varepsilon}^{y}(t), K(s)\right) \geqslant \delta\right) v_{\varepsilon}(d y) \\
= & \int_{E} \mathbb{P}\left(\operatorname{dist}_{E}\left(u_{\varepsilon}^{y}(t), K(s)\right) \geqslant \delta, u_{\varepsilon}^{y} \in H_{s, \delta}(\bar{n})\right) v_{\varepsilon}(d y) \\
& +\int_{E} \mathbb{P}\left(\operatorname{dist}_{E}\left(u_{\varepsilon}^{y}(t), K(s)\right) \geqslant \delta, u_{\varepsilon}^{y} \notin H_{s, \delta}(\bar{n})\right) v_{\varepsilon}(d y),
\end{aligned}
$$

where $\bar{n}$ is the integer found in Lemma 7.4 (see also the proof of Theorem 7.3). Then, due to Lemma 7.4 and to the upper bounds for the trajectories of $u_{\varepsilon}^{y}$ proved in [3, Theorem 6.3], we can fix $\varepsilon_{1}>0$ such that for any $\varepsilon \leqslant \varepsilon_{1}$

$$
v_{\varepsilon}\left(\left\{x \in E ; \operatorname{dist}_{E}(x, K(s)) \geqslant \delta\right\}\right) \leqslant \exp \left(-\frac{s-\gamma / 2}{\varepsilon^{2}}\right)+\int_{E} \mathbb{P}\left(\operatorname{dist}_{E}\left(u_{\varepsilon}^{y}(t), K(s)\right) \geqslant \delta ; u_{\varepsilon}^{y} \notin H_{s, \delta}(\bar{n})\right) v_{\varepsilon}(d y) .
$$

Then we can conclude the proof of the theorem, by using the same arguments used in the proof of Theorem 7.3.

\section{Acknowledgements}

The first named author would like to warmly thank Irena Lasiecka for many useful and interesting discussions about the control problem in Section 4 and the proof of Lemma 3.5. She also would like to thank Philippe Clément and Jerzy Zabczyk for suggestions about Proposition 2.1 and Theorem 4.2 respectively.

The second named author would like to thank his hosts at several stays at the Scuola Normale Superiore in Pisa.

\section{References}

[1] S. Cerrai, Second Order PDE's in Finite and Infinite Dimension. A Probabilistic Approach, Lecture Notes in Mathematics, vol. 1762, Springer-Verlag, Berlin, 2001.

[2] S. Cerrai, Stochastic reaction-diffusion systems with multiplicative noise and non-Lipschitz reaction term, Probab. Theory Related Fields 125 (2003) 271-304.

[3] S. Cerrai, M. Röckner, Large deviations for stochastic reaction-diffusion systems with multiplicative noise and non-Lipschitz reaction term, Ann. Probab. 32 (2004) 1-40.

[4] Ph. Clément, G. Sweers, Uniform anti-maximum principles, J. Differential Equations 164 (2000) 118-154.

[5] E.B. Davies, Heat Kernels and Spectral Theory, Cambridge University Press, Cambridge, 1989.

[6] I. Daw, Principe de grandes déviationes pour une mesure invariante associée à un processus de diffusion en dimension infinie, Ph.D. Thesis, University of Rouen (1998).

[7] M.D. Donsker, S.R.S. Varadhan, Asymptotic evaluation of certain Markov process expectations for large time, I, Comm. Pure Appl. Math. 28 (1975) 1-47;

M.D. Donsker, S.R.S. Varadhan, Asymptotic evaluation of certain Markov process expectations for large time, II, Comm. Pure Appl. Math. 28 (1975) 279-301;

M.D. Donsker, S.R.S. Varadhan, Asymptotic evaluation of certain Markov process expectations for large time, III, Comm. Pure Appl. Math. 29 (1976) 389-461.

[8] W.G. Faris, G. Jona-Lasinio, Large fluctuation for a non linear heat equation with noise, J. Phys. A 15 (1982) 3025-3055.

[9] M.I. Freidlin, Random perturbations of reaction-diffusion equations: the quasi deterministic approximation, Trans. Amer. Math. Soc. 305 (1988) 665-697.

[10] M.I. Freidlin, A.D. Wentzell, Random Perturbations of Dynamical Systems, Springer-Verlag, Berlin, 1984.

[11] S. Peszat, Large deviation estimates for stochastic evolution equations, Probab. Theory Related Fields 98 (1994) 113-136.

[12] T. Runst, W. Sickel, Sobolev Spaces of Fractional Order, Nemytskij Operators and Nonlinear Partial Differential Equations, Walter de Gruyter, Berlin, 1996. 
[13] R. Sowers, Large deviations for a reaction-diffusion equation with non-Gaussian perturbation, Ann. Probab. 20 (1992) $504-537$.

[14] R. Sowers, Large deviations for the invariant measure of a reaction-diffusion equation with non-Gaussian perturbations, Probab. Theory Related Fields 92 (1992) 393-421.

[15] H. Triebel, Interpolation Theory, Function Spaces, Differential Operators, North-Holland, Amsterdam, 1978.

[16] S.R.S. Varadhan, Asymptotic probabilities and differential equations, Comm. Pure Appl. Math. 22 (1969) $261-286$. 